\title{
Zur Katalyse durch Chromsäure und ihre Salze.
}

\author{
II. Mitteilung. \\ Von \\ EUGgin SPITALSKr.
}

Mit 10 Figuren im Tert.

Im folgenden soll ein höchst eigentỉmlicher Reaktionsverlauf beschrieben werden, welchen ich bei meinen Studien uber die katalytische Zersetzang des Wasserstoffsuperoxyds durch freie Chromsäure beobachtet habe. Ein solcher oder ähnlicher Fall von isothermer Reaktionskinetik ist meines Wissens weder bis jetzt beobachtet worden, noch theoretisch vorausgesehen. Dieser Fall dürfte vielleicbt nicht ohne Interesse sein, einerseits in Anbetracht der noch immer diskutierten Frage über die Analogie resp. den Unterschied zwischen gewöhnlichen chemischen Reaktionen (zumal in homogenen Systemen) und sogenannten Fermentreaktionen, andererseits hinsichtlich der Theorie der Zwischenstufen bei chemischen Vorgängen.

Mit der weiteren Ausarbeitung der zu beschreibenden Reaktion speziell auch ihrer Theorie und mit Aufsuchung analoger Falle bin ich noch beschäftigt, jetzt möchte ich wegen Änderung meiner äuf́seren Dmstände vorläufig nur das Tatsachenmaterial mitteilen, welches die Erscheinung selbst wiedergibt.

Die Anwesenheit von relativ erheblichen Mengen $H^{-}-$Ion, wodurch sich die Dichromsäure von ibrem Neutralsalze, dem Dichromat unterscheidet, ${ }^{1}$ ändert die Einwirkung auf das Wasserstoffsuperoxyd ganz aufserordentlich.

Unter Einwirkung des Kaliumdichromats ist die Zersetzung des Wasserstoffsuperoxyds eine rein katalytische Reaktion mit einem

1 Siehe: Utber den Zustand der Chromstiure und der Chromate in wässeriger Lösung, Z. anorg. Chem. 54 (1907), 265. Es seien hier einige Druckfehler der dortigen Abhandlang berichtigt: auf Seite 275 Anmerkung anstatt: prakt. Ohom. lies: phys. Chem.; auf 8 . 275 Zeile to v. u. anstatt: molarerer lies: molarer; auf $\mathrm{S} .277$ Tabelle anstatt: $10^{4}=$ lies: $10^{4} . k=$; auf S. 311 Anmerkung 1 anstatt; $\frac{0.456^{8}}{2}$ lies: $0.456^{8}$; auf S. 314 Zeile 11 v. 0 . anstatt: ungeftihr gleich lies: ungefahr. 
monomolekularen Reaktionsverlaufe. ${ }^{\mathrm{I}}$ Da die Chromsäure durch das Wasserstoffisuperoxyd zum Teil zu Chromion reduziert wird, kơnnte man erwarten, dafs die Einwirkung freier Chromsäure auf das Wasserstoffauperoxyd einerseits in der katalytischen Zersetzung desselben, andererseits in der nebenhergehenden Reduktion der Chromsuture selbst bestehen wird, so dals man hier eine Katalyse mit einer den Katalysator zerstörenden Nebenreaktion baben wird. Des wollte sich in einem Abfall der Geschwindig. keitskonstant orster Ordnung äufsern. Ws hat sich aber etwas ganz anderes gezeigt. Schon mit blofsem Auge kann man den Unterschied zwischen der in meiner ersten Mitteilung beschriebenen Dichromatkatalyse und der Wirkung freier Chromsăure sehen. Während dort beim Zusammenmischen der Lösungen sich das Reaktionsgemisch. unmittelbar braunrot färbte, dann diese Farbe mit dem Fortschreiten der Reaktion nach und nach verschwand und in die rotgelbe des Dichromates überging, färbt sich hier die Wasserstoffsuperoxydlösung bei Zugabe von wenig Chromaäure momentan blau, welche Farbe aber nach 1-2 Minuten verschwindet, um der für die katalytische Reaktion charakteristischen braunroten Platz zu machen, dieselbe bleibt lange Zeit bestehen, selbst ihre Intensität nimmt dabei kaum $\mathrm{ab}^{2}$, und dann wird beinahe plötzlich die Flizsuigkeit zuerst dunkler und unmittelbar darauf hellrotgelb, wie eine gewöhnliche Chromsäurelösung. Parallel mit allen diesen Farbenänderungen entwickelt sich Sauerstoff and in dem Momente, wodie Lösung wieder hellrotgelb wird, hört die Sanerstoffentwickelung auf.

Man kann diese Erscheinung zo einem schönen Demonstrationsversuche für eine Katalyse mit direkt sichtbaren Zwischenprodukten und insbesondere für den merkwürdigen eben geschilderten Reaktionsverlauf machen, wenn man etwas mehr Chromsäure (etwa $\mathbf{5}$ ccm einer 0.01 molaren Lösung) auf konzentriertes Wasserstoffsuperoxyd (etwa $20 \mathrm{ccm}$ einer $20-30 \%$ Lösung) einwirken lälst und die Flüssigkeit noch eine kurze Zeit auf $50-60^{\circ}$ erwärmt, bis die Reaktion sehr energitch wird. Dabei fängt die braunrote Flùssigkeit an zu kochen und:der Saverstoff strömt mit Wasserdampf zusammen

${ }^{1}$ Erste Mitteilung. Z. anorg. Chem. 53 (1907), 184. In dieser Abhandlung sind die Formeln $\mathrm{K}_{9} \mathrm{Cr}_{9} \mathrm{O}_{4}$ in den Tabellen 4 , 5 und 6 als $\mathrm{K}_{2} \mathrm{CrO}_{4}$ zu lesen. In Fig. 1 lies dort $\mathrm{H}_{2} \mathrm{O}_{2}$ statt $\mathrm{K}_{2} \mathrm{O}_{2}$.

Die zum Teil schon auggeführte quantitative kolorimetrische Untersucbung der Konzentration des Zwischenproduktes im Laufe der Reaktion wird, besonders auch an anderen Beispielen, fortgesetzt. 
aus dem kleinen Kölbchen heraus; dureh die weitere Solbsterhitzung wird die Reaktion immer stürmisober, die Stauerstoff- und Dampfentwickelung immer heftiger und plötzlich mit einem Sohlage hörti das Kochen und dis Gasentwickelung absolut auf und die Flassigkeit. wird gleichzeitig ebenso plbtzlich wieder hellrotgelb. Setzt man jetzt zu einer Probe dieser die Chromsäure onthaltenden Fluseigkeit. Schwefelsäure zu, dann zeigt sich keine Blaufărbung mehr, das Wasserstoffsuperoxyd ist also vollständig arsetzt. Gibt man: aber zu der hellrotgell gewordenen Lösung frisches, konzentriertes Superosyd, dann furbt sioh die Flatseigkeit wieder von neuem rotbrann. (ohne sich zuerst blau zu färben) und die Reaktion geht wieder wie vorhor. Das Interessanteste zeigt sioh aber: erst bei genauen: kinetischen Mesenngen: Sie wurden von mir genan so, wie mit den Chromaten ausgefuhrt, d. h. es warde dia Geschwindigkeiti der Sauerstoffentwickelung bei konetanter Temperatur $25^{\circ} \pm 0.02$ gemessen.

Analog dex Beobachtung, dals: die rotbranne Parbe der Lösung während der fortsohreitenden Realction maum schwächor wird, hat sich nun gezeigt, dals sich die Geschwindigkeit dev Gasentwickelung bei einem Überschusse an Superoxyd bis gegen Ende der Reaktion sehr wang andert, dann aber nach 1 bis 2. Stunden, wenn etwa $90 \%$ des Superoxyds schon zersetzt sind, beinahe plotalich axf das zwei- bis dreifache ateigt und dan e benso gchmell wieder fallt, um nach 5-10 Minuten gleich Noll. zu werden. Die luösung enthut dann kein Superoxyd mebr, was sohon: daraus zu sehen ist, dafs sich das der angewanten Superoxydmeage entspreckende Samerstoffolum entwickelt bat, welobes auch nach 2 Tagen keme Zunahme welir zoigt.

Von eimer Realition mit irgondwelchen einfach on kinetischen Ordnung kann hier natiliklich keine Piede sein. On diese meskwündige Reaktion wather zu untersuchen, habe ich mich daher vorlufig der graphisehen Darstellung bedienes molisen, indem ich entweder die entwickelte Bauerstoffinenge resp. den prozentischen Umatz als Funltion der Zeit, oder aber dive in kleimen Intervallen gemessene mittlere Reaktionsgeschwindigkeit (die pro: Zeiteinheit entwickelte Gasmengeresp. zersetzte Wasserstoffisuporoxydmenge) als Funktion der in der Lüsung noch vorhandenen Superoxydkonzentpation aufzeichnete. Um alle Versuche miteinander leieht verglevehbar zw

$1 Z$. anorg. Chem. 58, 184 . 
machan, lisbe: ich die Reaktínsgenchwindigkeit, d. h. die pro Zeiteinheit zersetzte Superoxydmenge ebenso wie seine jowails noek rorbsndene Konzentration in Molen pro Liter des Reaktionsgemisches ausgedrückt, während tatsllchlich je $20-32 \mathrm{ccm}$ des Gemisches sich in dem Verstahokölbehen befanden.

Ia den folgenden Tabollen und Figuren werden bedeuten:

$t=$ Zeit in Minuten;

$x=$ das zur Zeit $t$ direkt abgelesene Sauerstoffgasvolum in Kubikzentimetern bei der Temperatur $\vartheta^{0}$ und dem korrigierten Druck $p$;

$C_{1}$ bzw: $C_{2}=$ die daraus nach der Gleichung

$$
C_{1}=\frac{x \cdot 273 \cdot p \cdot 2}{(278+\vartheta) \cdot 760 \cdot 22,4 \cdot v}
$$

berectinete Superoxydmenge in Molen pro Liter des Reaktionsgemisches (bei $v$ ccm des Gemisches);

$C_{0^{\prime}}=$ die dem Endrolum des Gases entsprechende, durch. die Titration mit Kaliumpermanganat immer kontrollierte Menge des angowaten Superoxyds ebenso in Molen pro Liter;

$C_{2}^{\prime}-C_{1}=$ die zwischen zwei' aufeinanderfolgenden Zeitmomenten $t_{2}$ und $t_{1}$ zersetzte Superoxydmenge;

$\frac{C_{y}-C_{1}}{t_{y}-t_{1}}=$ die im Zeitmomente $\frac{t_{1}+t_{2}}{2}$ herrschende mittlere Reaktionsgeschwindigkeit.

$C=C_{0}-\frac{C_{1}+C_{2}}{2}=$ die in diesern Momente noch vorhandene mittlex Supowanthonentration.

$\frac{100 \cdot C_{1}}{\theta_{0}} \pm$ der provatische Umagtz im Momente $t$.

Thok der echeinbaren Kompliziertheit der Reaktion, haben sich die Vetcuabe alo sehr gut reproduzierbar gezeigt, so dals die

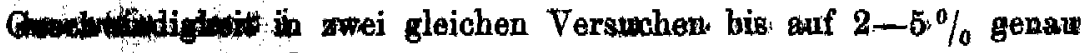
bestiment werden konnte. ${ }^{1}$

Um sowohl die Ausrechnungsweise zu zeigen, als den zeitlichen Verlanf einzelnar Versuche zu veranschauliction, filhre ich nachstehend zwai Beispiele vollständig an, während ich des weiteren die entsprechenden Kurven und die zu diesen nur unmittelbar gehörigen Zablen, oder sogar, wo die genaue Wiedergabe des allzugrofsen

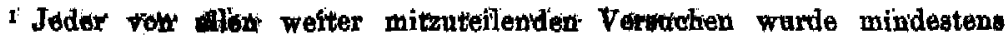
zweimal auggefubrt. 
Zahlenmaterials vor der Hand noch keine Bedeutung bätte, ${ }^{1}$ die Kurven allein benutzen werde.

Versuch 111.

\begin{tabular}{|c|c|c|c|c|c|}
\hline $\begin{array}{c}C_{\mathrm{H}_{8} \mathrm{C}} \\
{ }^{t} \\
\text { Minuten } \\
=====\end{array}$ & $\begin{array}{l}\mathrm{CCrO}_{2}= \\
=C_{0}=\end{array}$ & $\begin{array}{r}\text { fangskonz } \\
115 \text { Mol. } \\
\theta= \\
C_{1} \cdot 10^{8}\end{array}$ & $\begin{array}{l}\text { ration der Cl } \\
\text { Liter (nach } \\
\begin{array}{c}p \text { korr. }=7 \\
C_{2}-C_{1} \\
t_{2}-t_{1} \\
10^{5}\end{array}\end{array}$ & $\begin{array}{l}\text { romä̈ure }=0.0014 \\
\text { der Analyse mit } \mathrm{K} \\
6.5 \text {. } \\
\left(C_{0}-\frac{C_{1}+C_{2}}{2}\right)+10^{8}\end{array}$ & $\begin{array}{l}{\left[\mathrm{nO}_{4}\right) .} \\
\frac{10 \cdot C_{1}}{C_{0}} \\
==\end{array}$ \\
\hline 5 & 1.92 & 7.5 & 150 & 110.7 & 6.6 \\
\hline 10 & 8.64 & 14.2 & 75 & 106.8 & 12.4 \\
\hline 26 & 6.41 & 25.0 & 97 & 92.4 & 21.8 \\
\hline 40 & 9.72 & 37.9 & 94 & 76.9 & 83.1 \\
\hline 60 & 14.07 & 54.9 & 84 & 64.2 & 48.0 \\
\hline 86 & 18.93 & 73.8 & 67 & 44.0 & 64.5 \\
\hline 101 & 21.42 & 83.5 & 64 & 83.9 & 72.9 \\
\hline 111 & 23.22 & 90.5 & 70 & 27.5 & 79.0 \\
\hline 115 & 24.22 & 94.4 & 98 & 22.0 & 82.5 \\
\hline 122 & 26.77 & 104.4 & 143 & 15.1 & 91.2 \\
\hline 126 & 27.54 & 107.4 & 75 & 8.6 & 93.8 \\
\hline 129 & 28.04 & 109.3 & 69 & 6.1 & 95.5 \\
\hline 132 & 28.32 & 110.4 & 27 & 4.6 & 96.4 \\
\hline 138 & 28.53 & 111.2 & 13 & 3.7 & 97.1 \\
\hline 168 & 29.13 & 113.6 & B & 2.1 & 99.2 \\
\hline $12^{h}$ & 29.35 & 114.5 & & & 100 \\
\hline
\end{tabular}

(S. Versuch 195, S. 77.)

Um Platz zu sparen, habe ich auch in diesen Versuchen nicht alle Ablesungen angegeben, sondern nurvielleicht den dritten Teil, so dals die in der 4. Kolumne stehenden Werte der Geschwindigkeit $\frac{C_{2}-C_{1}}{t_{2}-t_{1}} \cdot 10^{5}$ nicht nur ans den hier angefiubrten Werten von $C$ und $t$ berechnet sind, sondern anch aus den noch dazwischen liegenden Ablesungen und ebenso die Werte von $C_{0}-\frac{C_{1}+C_{2}}{2}$ in der letzten Kolumne. Dasselbe gilt auch für die weiteren Tabellen. Aus diesen zwei ganz zufallig heranggegriffenen Beispielen sieht man schon die Eigentamlichkeit der Reaktion. Nachdem sich die Geschwindigkeit (Versuch 111) in 75 Minuten ( 26 bis 101) nur von $97 \cdot 10^{5}$ auf $64 \cdot 10^{5}$. geändert hat, wobei die Superoxydkonzentration von 0.092 auf

- Oder, wo besondere Ứbersichtstabellen die herrorzuhebenden Regelmälsigkeiten erlüutern werden. 
Versuch 195.

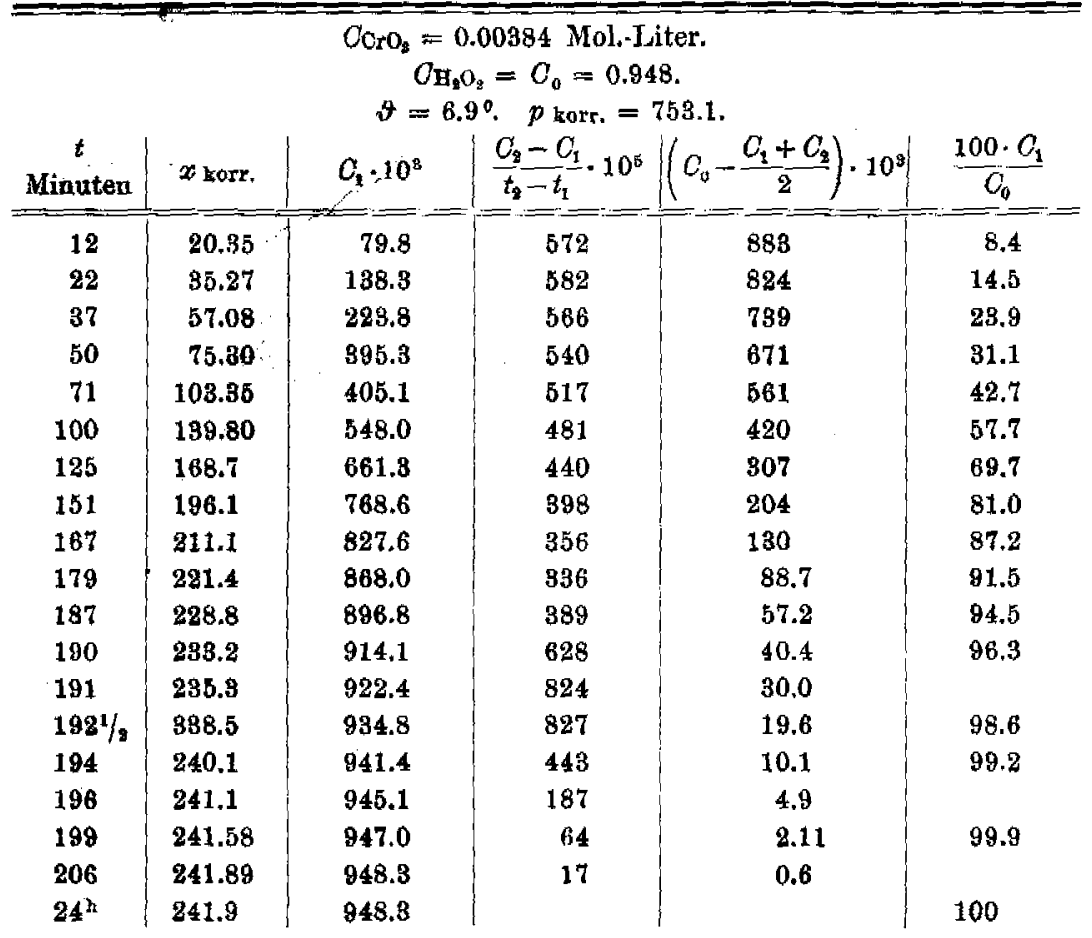

0.084 Mol. pro Liter sinkt, steigt die Geschwindigkeit dann innerhalb 10 Minuten von $70 \cdot 10^{5}$ auf $143 \cdot 10^{8}$, um dann in 7 Minuten wieder auf $69 \cdot 10^{5} \mathrm{zu}$ fallen und nach weiteren 10 Minuten hört die Reaktion praktisch auf, wobei in der Lösung noch kaum $1 \%$ des angewandten Superoxyds ubrig geblieben ist.

Noch auffallender ist die Erscheinung im Versuche 195, wo eine grölsere Superoxydmenge ron Anfang an genommen wurde. $21 / 2$ Stunden lang gebt die Reaktion mit einer Gasentwickelungsgeschwindigkeit von etwa $1-1 \frac{1}{2} \mathrm{ccm}$ pro Minute, die Geschwindigkeit vermindert sich nicht einmal auf die Hälfte, während die Substratkonzentration (d. b. die $\mathrm{H}_{2} \mathrm{O}_{2}$-Konzentration) etwa a uf den zehnten Teil sinkt und dann, nachdem schon $92 \%$ der angewandten Superoxydmenge zersetzt sind, springt die Geschwindigkeit in 12 Minaten bereits an mehr als das $z$ weifache, und nach weiteren 8 Winiten ist die Reaktion am Ende, indem $99.9 \%$ des Superoxyde zersetats sind. Fig. 1 macht diese Verbältnisse anschaulich, Abszissen sind die Zeiten, Ordinaten die zugehörigen Reaktionsgeschwindigkeiten. 
Anfangakonzentration.

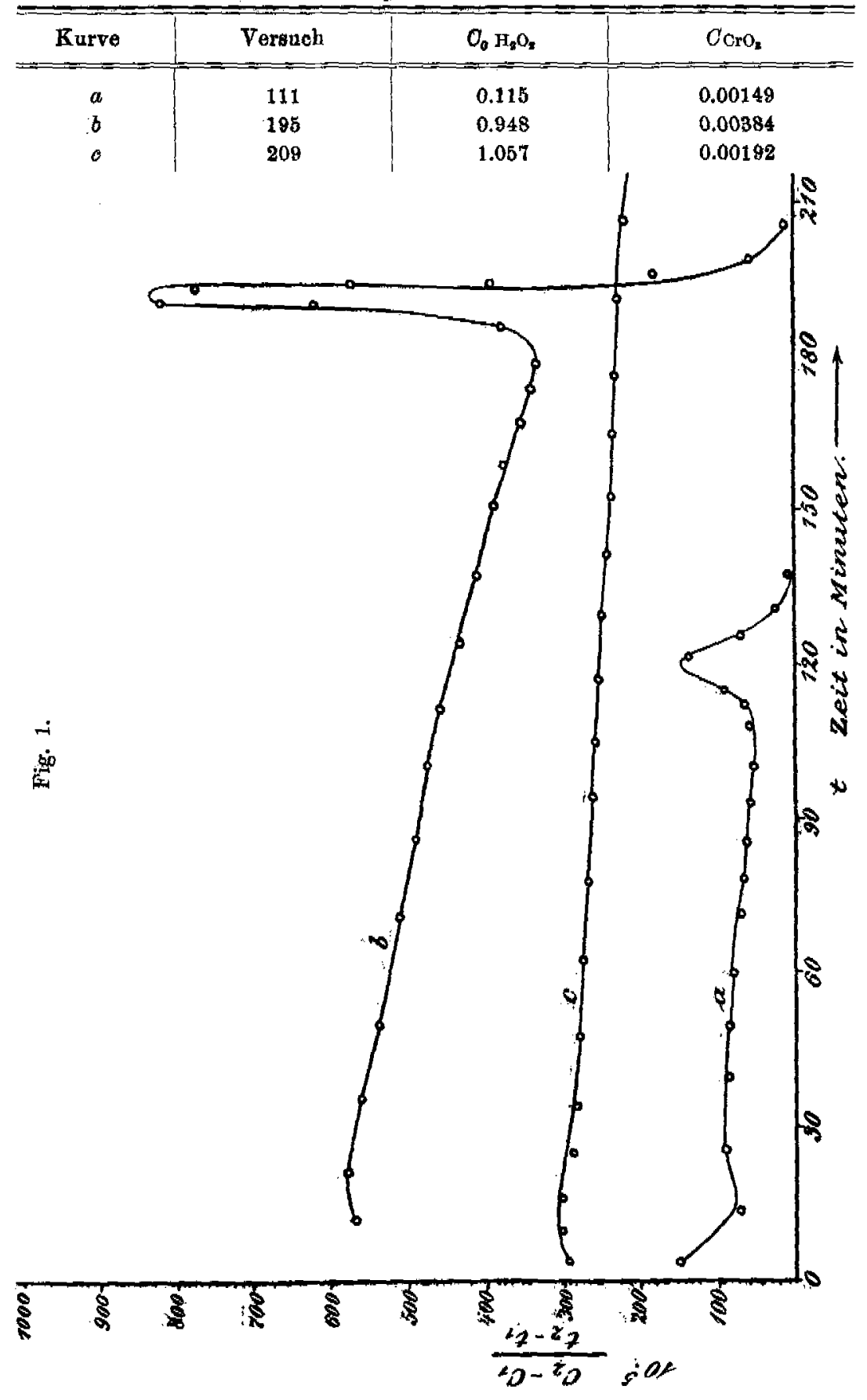


Abgesehen von den Unregelmälaigkeiten am Anfang der Kurven, - wigen diese :eizen sahr gleichmälsigen Gang. ${ }^{1}$

Die Kurve a, welche wagen Platzmangels nur zum Teil aufgezeiahnøt i st, ${ }^{2}$ gehört zn einon Tersuche mit den Anfangskonzentrationen $C_{\mathrm{CrO}_{3}}=0.00192$ und $C_{\mathrm{B}_{3} \mathrm{O}_{3}}=1.057 \mathrm{Mol}$. Der hier stehende Teil zeigt, wie wenig die Geschwindigkeit bei einem sebr grofsen Überschusee an Superoxyd mit der Zeit abnimmt, obwohl sich die Konzentration des letzteren in dem hier angefuhten Teile schon von 1.057 auf 0.510 geändert hat. Dieser Versuch ging noch 240 Minuten in demselben Tempo weiter, und dann nach 8 Stunden im Ganzen, als nur noch 0.039 Mol. pro Liter $\mathrm{H}_{2} \mathrm{O}_{2}$ unzersetzt geblieben waren (etwa $4 \%$ der anfänglichen Menge), stieg die Geschwindigkeit innerhalb 10 Minuten auf das dreifache, um gleich darauf auf Null zu fallen.

Aus der Fig. 1 bekommt man natürlich keine Übersicht uber die Abhängigkeit des Reaktionsverlaufes von den angewandten Konzentrationen, welche man äberbaupt erst dann sehen kann, wenn man die Reaktionsgeschwindigkeit nicht als Funktion der Zeit, mandenn' der Superoxydkonzentration betrachtet. Fig. 1 sollte nur zunïchst den,allgemeinen zeitlichen Reaktionsverlauf zeigen.

\section{Variable Anfangakonzentration des Wasserstoffeuperoxyds bei koustanter Chromsäurekonzentration.}

Es : wurde munehr systematisch untersucht, wie der Reaktionsverlax von der cangewandten Wasserstoffsuperoxydkonzentration bei ikanatanter. Anfangskonzentration der Cbromsäure abbängt. Da eber, wie gesagt, ein Teil der Chromsăure dabei reduziert wird, so war auerst nachzusehen, wie diese Reduktion von der angewandten Superoxydmenge abhängt. Ware die Redultion nur eine, gewöhnliche Nebenreaktion, dann mülste der am Fnderdor Wanerotoffeuperoxydzersetzung reduzierte Teil mit der Antangskengentration des $\mathrm{H}_{2} \mathrm{O}_{2}$ resp. mit der Dauer des Versuches steigen.

1. Die Unzegelmaraigkeiten in den ersten 5-10 Minuten kommen daher, dafs die in den Fallröhrchen befindliche Katalyastorlösung nach dem Einfallen des Röhrchens in die Wassersuperoxydlöstung sich nicht momentan mit derselben vollotrindig vermigent.

4. In eiver enderan. Abhängigkeit wird diesen Verench noch weiter (Fig. 8) unten vorkommen. 
Die Versuche haben aber gezeigt, dafs derselbe von der Superoxydanfangskonzentration und, in Prozent der angewandten Chromsäurekonzentration ausgedrückt, ebenso von dieser letzteren beinahe unabhängig ist. (Tabelle 1.)

Tabelle 1.

\begin{tabular}{|c|c|c|}
\hline \multicolumn{2}{|c|}{ Anfangekonzentration } & \multirow{2}{*}{$\begin{array}{l}\text { Reduzierter Tei } \\
\text { der Chromsäure } \\
\text { in } \%\end{array}$} \\
\hline des $\mathrm{H}_{2} \mathrm{O}_{2} \quad \mathrm{CH}_{2} \mathrm{O}_{2}$ & der Chromeäure $\mathrm{CrrO}_{\mathrm{s}}$ & \\
\hline 0.225 & 0.00384 & 25 \\
\hline 0.225 & 0.00384 & 25 \\
\hline 0.450 & 0.00384 & 26 \\
\hline 8.50 & 0.00884 & 28 \\
\hline 0.291 & 0.0051 & 28 \\
\hline 0.582 & 0.0051 & 28 \\
\hline 1.165 & 0.0051 & 29 \\
\hline 0.582 & 0.0102 & 29 \\
\hline
\end{tabular}

Daraus geht hervor, dals die Reduktion der Chromsäure, wenn sie auch als eine Nebenreaktion stattfindet, jedenfalls viel schneller vor sich geht, als die katalytische Zersetzung des Wasserstoffsuperoxyds, und dafs alles, was von der Chromsäure bei ihrer gegebenen Konzentration (ohne überschüssige fremde Saure) durch einen Überschuls von Wasserstoffsuperoxyd reduziert werden kann, in allen in der Tabelle 1 angeführten Versuchen mit verschiedenen Anfangskonzentrationen des $\mathrm{H}_{2} \mathrm{O}_{2}$ auch tatsăchlich immer und unabhängig von der Zeitdauer der Hauptreaktion $d$. h. der Katalyse reduziert wird. Es liegt nahe zu vermuten, dafs diese Reduktion sogleich nach dem Zusammenmischen der Lösungen (Blaufärbung) stattfindet, so dafs alle Versuche mit gleicher Anfangskonzentration der Chromsänre auch gleiche katalytisch aktive (unreduzierte) Menge des Katalysators haben, was durch besondere weiter unten zu besprechende Versuche noch wahrscheinlicher gemacht werden wird.

Entsprechend der Tatsache, dafs die Geschwindigkeit der Wasserstoftsuperoxydzersetzung, abgesehen von ihrem am Ende der Reaktion stattfindenden Hinaufsteigen, sich sehr wenig mit der Konzentration des $\mathrm{H}_{2} \mathrm{O}_{3}$ andert, hat sich bei den kinetischen Versuchen zunächst gezeigt, dafs bei gleicher Katalysatormenge der relative Umsatz in Prozenten der angewandten Wasser. stoffsperoxydmenge in gleichen Zeiten um so kleiner ist, je gröfser die angewandte Superoxydmenge war. ${ }^{1}$ In der

1 Wie wir im Schlufakapitel sehen werden, liegt hierin eine auffallende Analogie mit manchen Fermentreaktionen. 
Fig. 2 und Tabelle 2 sind die diesbezïglichen Resultate zusammengestellt.

Tabelle 2.

\begin{tabular}{|c|c|c|c|c|c|c|}
\hline $\begin{array}{l}\text { Ver- } \\
\text { such } \\
\vdots\end{array}$ & $\begin{array}{l}\text { Kurve } \\
\text { Fig. } 2\end{array}$ & $O \mathrm{CrO}_{3}$ & $O_{\mathrm{H}_{2} \mathrm{O}_{2}}$ & $\begin{array}{c}\text { Umsatz } \\
\text { in } \% \\
\text { nach } 10 \mathrm{Min} .\end{array}$ & $\begin{array}{c}\text { Umaatz } \\
\text { in } \% \\
\text { nach } 30 \mathrm{Min} .\end{array}$ & $\begin{array}{c}\text { Umsatz } \\
\text { in } \% \\
\text { nach } 70 \mathrm{Min} .\end{array}$ \\
\hline 114 & $a$ & 0.00298 & 0.049 & 96 & & \\
\hline 110 & $b$ & 0.00298 & 0.115 & 24 & 62 & \\
\hline 105 & & 0.00298 & 0.117 & 24 & 60 & \\
\hline 113 & $c$ & 0.00298 & 0.240 & 12 & 34 & 74 \\
\hline 109 & $d$ & 0.00288 & 0.498 & 6 & 20 & 47 \\
\hline 118 & & 0.00884 & 0.178 & 26 & 64 & \\
\hline 137 & & 0,00384 & 0.390 & 11 & 34 & 72 \\
\hline 195 & & 0,00384 & 0.948 & 7 & 19 & 42 \\
\hline
\end{tabular}

Die Zahlen der vierten und der drei letzten Kolumnen sind nicht genau umgekehrt proportional, weil die Reaktionsgeschwindigkeit doch nicht ganz unabhängig von der vorhandenen Wasserstoffsuperoxydkonzentration ist.

Trägt man die Versuchsergebnisse in ein Koordinatensystem (Fig. 3) mit der Ordinate „Realtionsgeschwindigkeit" und der Abszisse "noch vorhandene $\mathrm{H}_{2} \mathrm{O}_{2}$-Konzentration" ein, dann sieht man zuerst, dafs die Geschwindigkeit ähnlich wie in Fig. 1 mit abnehmender $\mathrm{H}_{2} \mathrm{O}_{2}$-Konzentration relativ nur sehr wenig bis zu einem Minimum abnimmt, worauf sie dann sehr steil bis zu einem scharf ausgeprägten Maximum steigt und sehr schnell wieder fallt. Die Abnahme der Geschwindigkeit mit der $\mathrm{H}_{2} \mathrm{O}_{2}$-Konzentration im nahezu horizontalen Teile der Kurven lälst sich ungefähr durch die Beziehung

$$
-\frac{d c}{d t}=k \cdot c^{1 / s}
$$

ausdrucken, d. h. die Reaktionsgeschwindigkeit ist ungefähr proportional der dritten Wurzel aus der Substratkonzentration. Diese Formel gilt jedoch nun angenähert, indem die Geschwindigkeit, wo sie sich dem Minimum nähert, noch weniger abnimmt, als es der obigen Formel entspricht. Das zeigt, dafs die wahre Abhängigkeit eine kompliziertere ist.

Aus dem Vergleiche verschiedener Versuche mit gleicher Konzentration der Chromsäpre geht jedoch hervor, dals es jedenfalls eine eindeutige Beziehung zwischen der Geschwindigkeit und der Z. anorg. Chèn. Bd. 66 , 


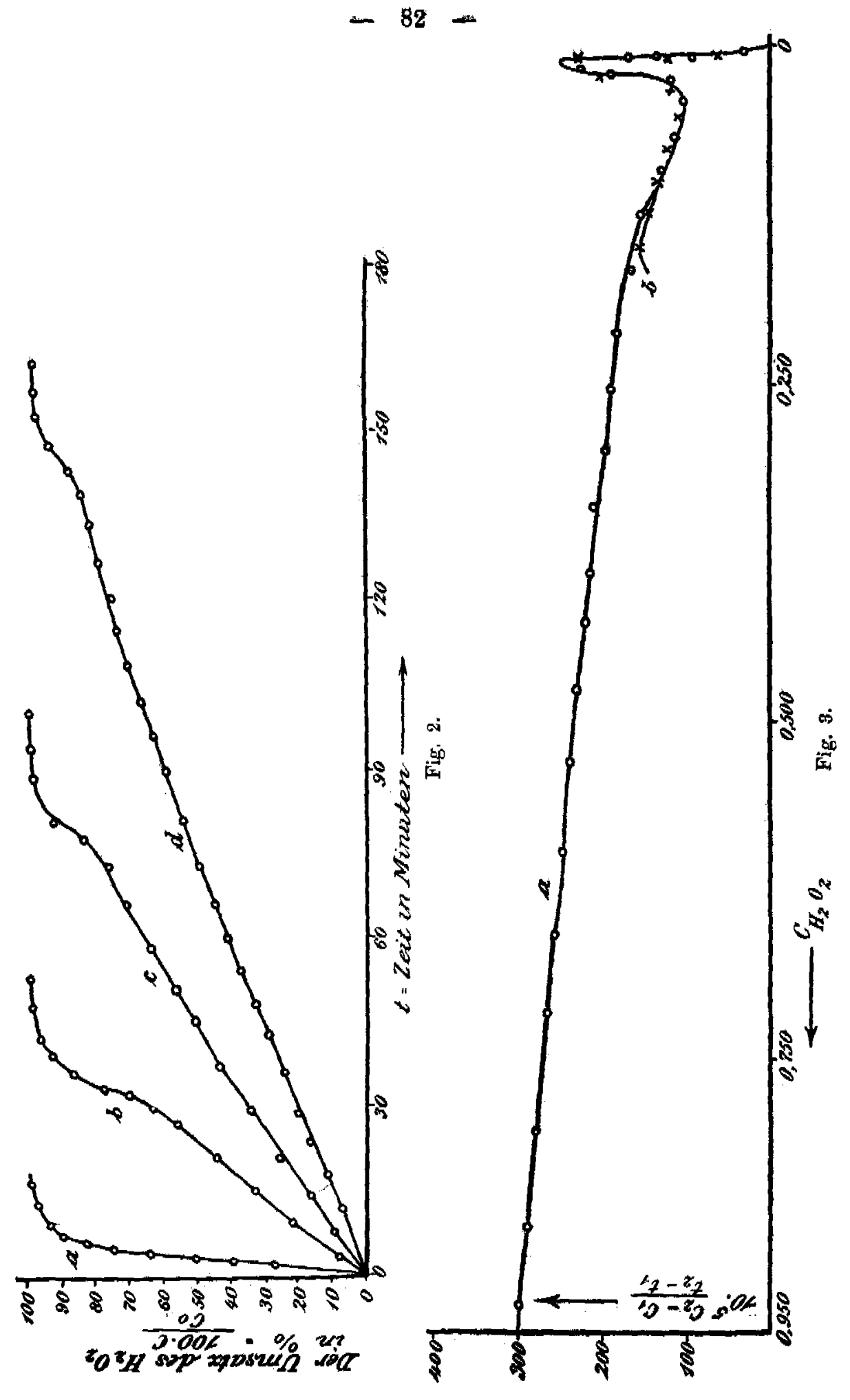




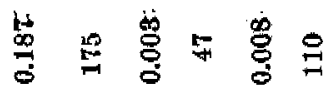

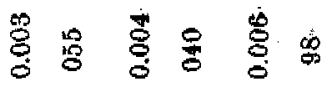

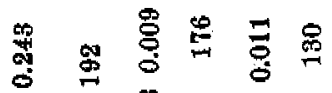

产 总

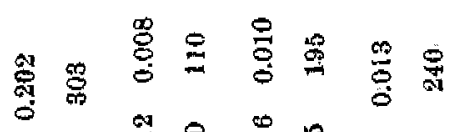

露

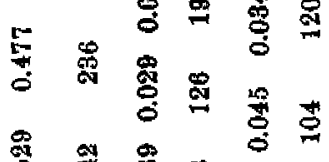

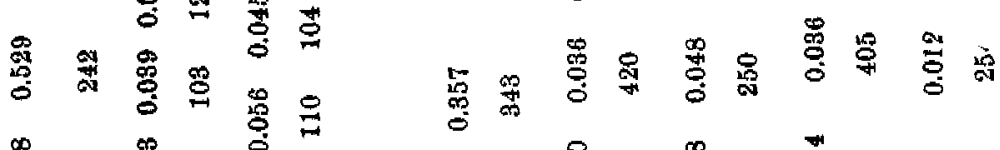

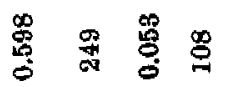

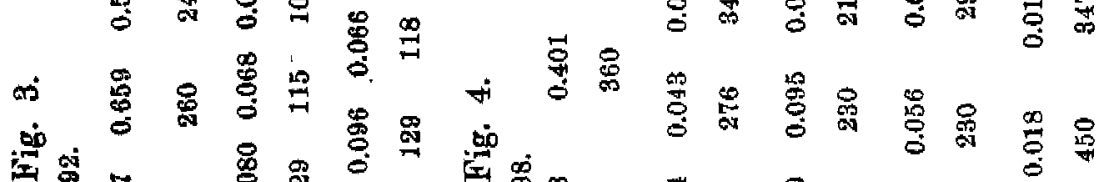

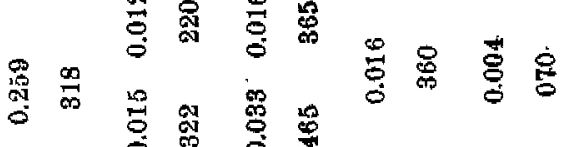

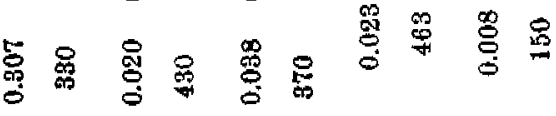

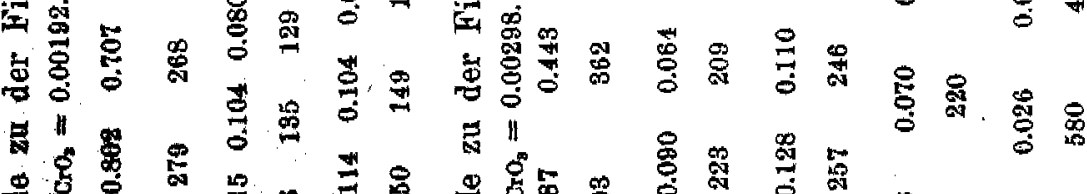

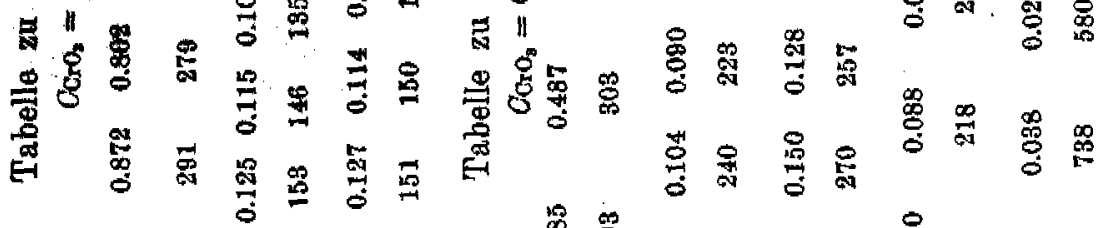

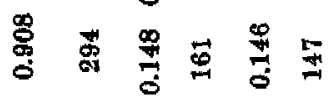

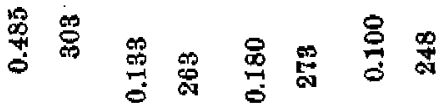

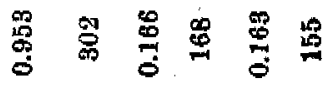

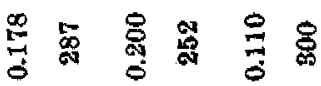

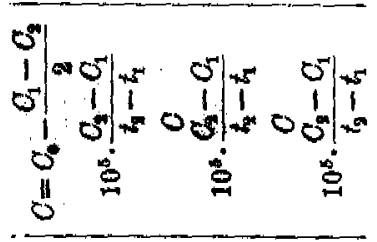

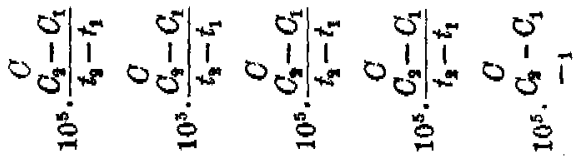

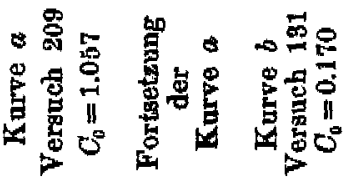

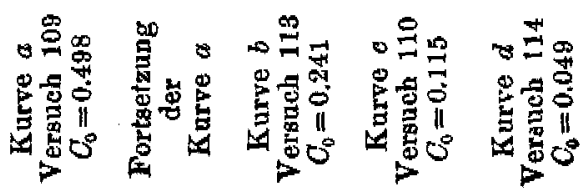




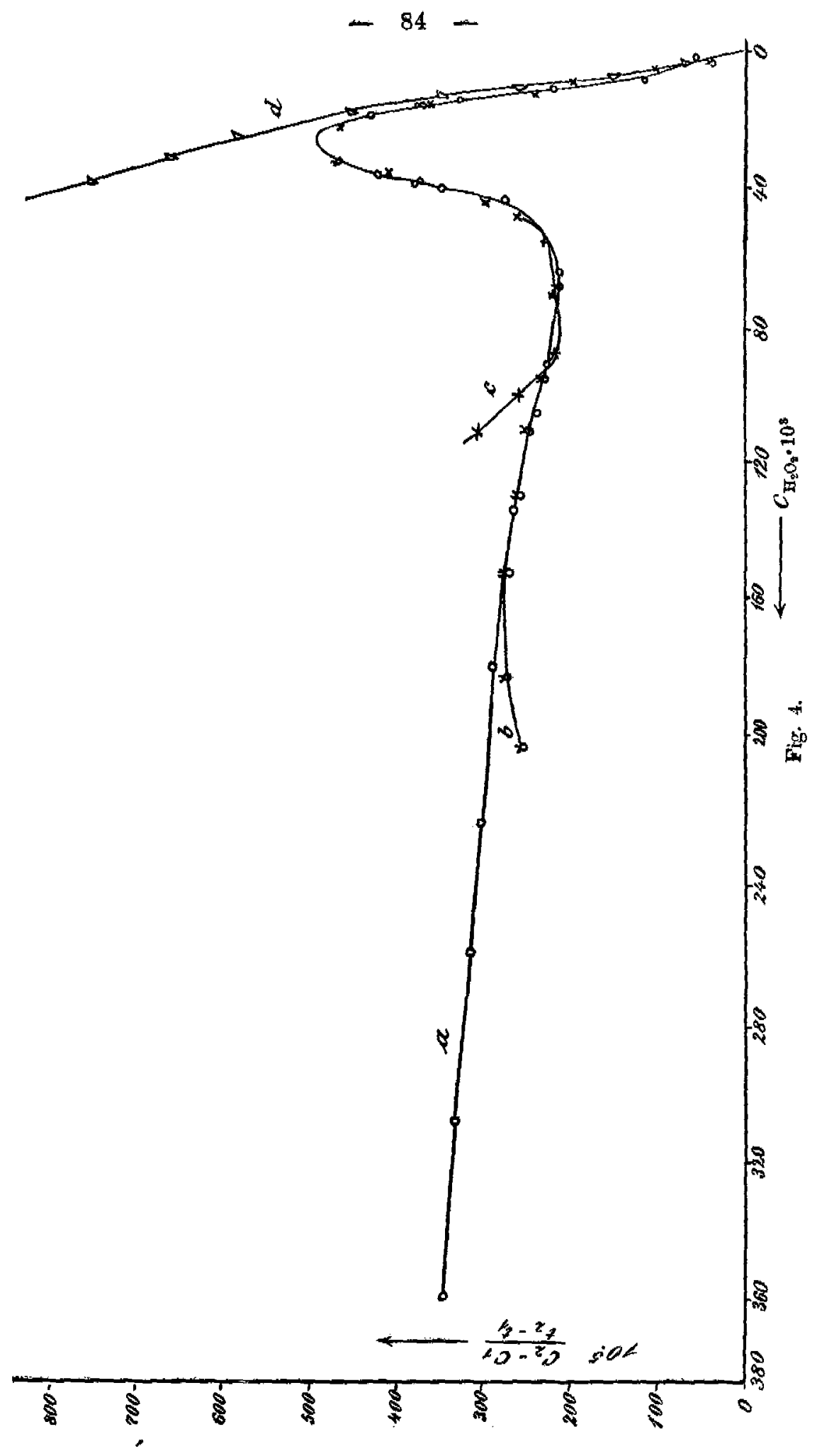


vorhandenen Wasserstoffsuperoxydkonzentration gibt, $d, b$, dafs $z u$ einer jeden Wasserstoffsuperoxydkonzentration eine bestimmte Reaktionsgeschwindigkeit gehört unabbängig von der anfänglichen Superoxydmenge. In den Figg. 3 und 4 sind mehrere Versuche mit verschiedener Anfangskonzentration des $\mathrm{H}_{2} \mathrm{O}_{2}$, aber mit je gleicher $\sigma_{\mathrm{CrO}_{2}}$ aufgetragen.

Man sieht, dafs die Kurven mit kleineren Anfangskonzentrationen des Wasserstoffsuperoxyds $(c, b)$ (nach tunwesentlichen Unregelmälsigkeiten am Anfang ${ }^{1}$ ) mit den Kurven, welche schon-die fünf-bis sechsfache Menge des zersetzten Superoxydes anzeigen, vollständig zusammenfallen. Versuche $a$ und $b$, Fig. 3 , welche nacheinander in sehr verschiedenen Zeiten mit verschiedenen Präparaten sowohl des $\mathrm{H}_{2} \mathrm{O}_{2}$ als der Chromsäure gemacht wurden, zeigen z. B. die Übereinstimmung innerhalb 2-3\% der Geschwindigkeiten und ebenso alle anderen. Die Übereinstimmung ist nicht nur im horizontalen Teile der Kurven eine gute, sondern auch in dem plötzlichen Anstieg am Ende, - wenn auch die Versuchsfehler in diesem Abschnitte der Kurven etwas grölser sind, als in dem ersten Teile, was an der Messung einer grolsen sich sehr schnell ändernden Geschwindigkeit in kleinen Zeitintervallen (alle Minuten) liegt. ${ }^{2}$ Kurve $d$, Fig. 4, zeigt einen Versuch mit einer Anfangskonzentration des Wasserstoffsuperoxyds, welohe nicht viel grölser war, als diejenige, bei welcher das Maximum der Geschwindigkeit auftritt. Wegen der auch bei der Reduktion stattfindenden Gasentwickelung fällt die Anfangsgeschwindigkeit etwas zu grofs aus, nimmt aber gleich ab und dann fällt diese Kurve mit den letzten absteigenden Ästen anderer Kurven zusammen.

Aus allen diesen Versuchen kann man schliefsen:

Bei gegebener Konzentration der Chromsäure:

Die Geschwindigkeit ist in jedem Momente definiert durch die rorbandeve Konzentration des Wasserstoffsuperoxyds.

Die Konzentration des Wasserstoffsuperoxyds, bei welcher das Minimum und das Maximum der Geschwindig.

2 Siebe Anm. 1 S. 79.

2 Durch bezondere Versuche habe ich mich uberzengt, dafs die Schüttelgescbwindigkeit des Apparates (300 Touren pro Minute) genügend grofs war, um keinen weiteren Einflufs auf die Gasentwickelung zu Laben. Vergl, die erste Mitteilung. Z. anorg. Chem. 53, 184. - WAltoN, Zeitschr. phys, Ohem. $47(1904), 185$, 
keit auftreten, ist eine bestimmte und von der Anfangskonzentration des Superoxyds unabhängige.

Daraus folgt unter anderem, dals die teilweise Reduktion der Chromsăure in ihrem zeitlichen Verlanfe keinen störenden Einflufs auf die katalytische Zersetzung des Wasserstoffsuperoxyds ausübt. Die oben ausgesprochene Vermutung, dafs diese Reduktion sogleich nach dem Zusammenmischen der Lösungen sehr schnell stattfindet, gewinnt sehr an Wahrscheinlichkeit. Man kann sich denken, dafs bei allen obigen Versuchen gleich am Anfang der Reaktion stets ein gleicher Bruchteil (vergl. Tabelle 1 Seite 80) der Chromsâure reduziert wird und der zurückgebliebene Teil weiter katalytisch wirkt. Dafs diese Wirkung nicht eine einfache ist, geht aus der Gestalt der Kurven hervor. Jedenfalls muls aber der Zustand des Katalysators in allen angefuhrten Versuchen unabhängig ron den urspringlichen $\mathrm{H}_{2} \mathrm{O}_{3}$-Konzentrationen der gleiche und in jedem Augenblicke der Katalyse durch die jeweilige $\mathrm{H}_{2} \mathrm{O}_{2}$ Konzentration eindeutig bestimmt sein.

Es war interessant, noch nachzusehen, welchen Einflufs auf die katalytische Wirksamkeit der Chromsäure der am Ende der Reaktion stattfindende Anstieg der Geschwindigkeit ausübt, d. b. ob hier nicht irgendwelche irreversible Änderungen in dem Zustande des Katalysators vorliegen. Um das zu entscheiden, habe ich zu dem Reaktionsgemische $(22 \mathrm{ccm})$ mit den Anfangekonzentrationen $C_{B_{3} O_{2}}=$ 0.916 und $C_{\mathrm{Cr}_{3}}=0.00384 \mathrm{Mol}$. pro Liter, nachdem alles Wasserstoffsuperoxyd sicher zersetzt worden war, eine frische Portion desselben (2 ccm einer $30 \%$ igen $\mathrm{H}_{2} \mathrm{O}_{2}$.Lösung) zugegeben und den Reaktionsverlauf wieder verfolgt. Dann wurden zu diesem Gemische nach Beendigung der Reaktion wieder $2 \mathrm{ccm}$ der Superoxydlősung gegeben und die Reaktion zum drittenmal in derselben Flussigkeit gemessen.

Fig. 5 gibt diese Versuche wieder. Die Kurven $a, b$ und $e$ verlaufen ganz parallel. Die Geschwindigkeitswerte von $b$ und $c$ sind nur etwas, d. h. annähernd proportional der durch die Neuzugabe der $\mathrm{H}_{2} \mathrm{O}_{\mathbf{a}}$ Lösung statttindenden Verdü nnung (das erste Mal von $22 \mathrm{ccm}$ auf 24, das zweite $\mathrm{Mal}$ anf $26 \mathrm{ccm}$ ) der Chromsäure vermindert. Zur Kontrolle wurde noch ein Normalversuch (Kurve $d$ ) mit einer frischen Chromsäurelösung gemacht, indem $\mathrm{zu}$ dem iublichen Reaktionsgemisch $(20 \mathrm{ccm}$ $\mathrm{H}_{2} \mathrm{O}_{2}$ und $2 \mathrm{ccm}$ Chromsäurelösung) noch $4 \mathrm{ccm}$ Wasser zugegeben wurden, so dafs die Verdinnung der genommenen Chromsäure von vornherein derjenigen im Versuch $c$, Kurve o, gleich war. Inner. halb der Versuchsfehler sind die Geschwindigkeitswerte in diesen Ver* 


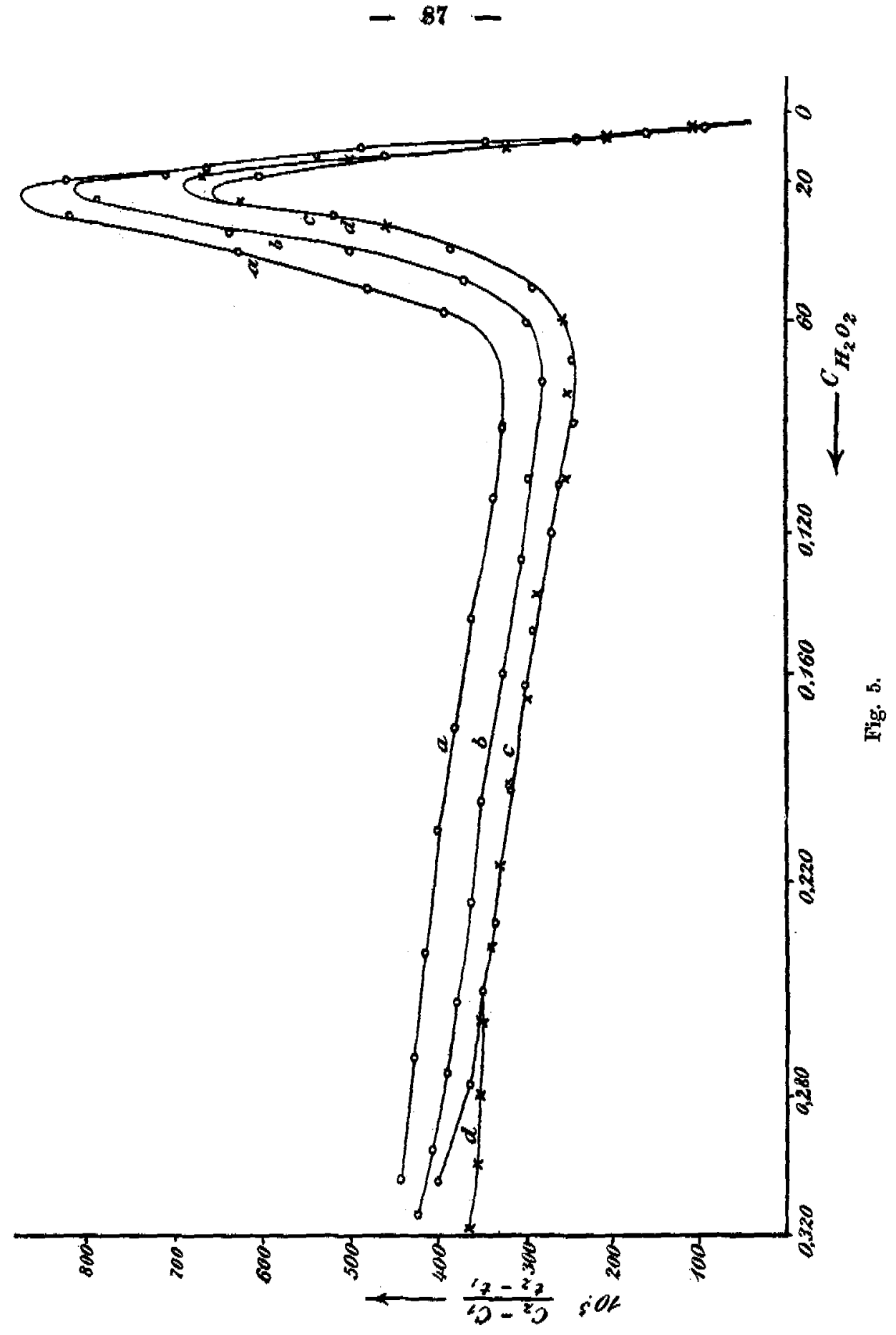




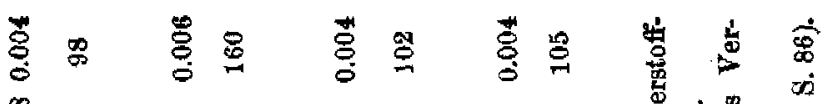

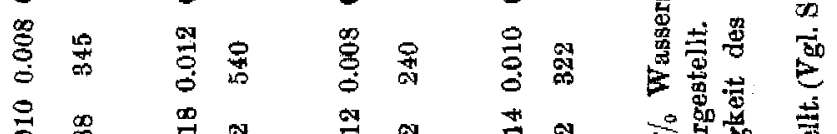

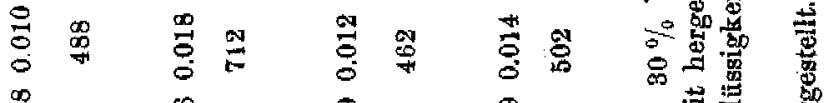

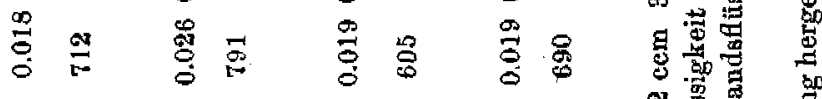

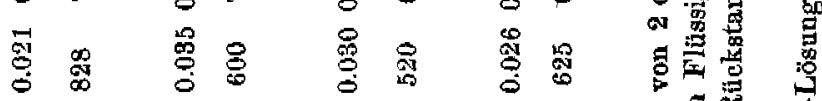

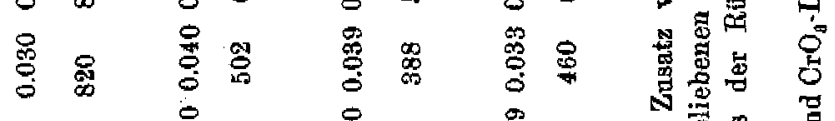

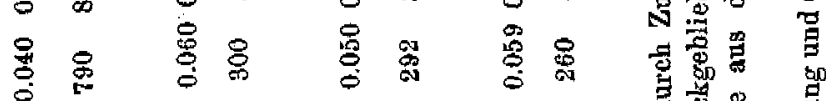

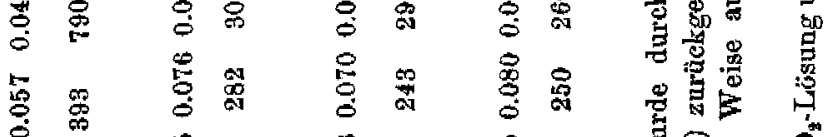

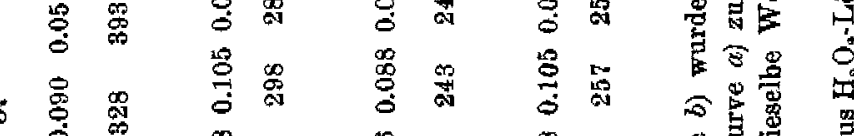

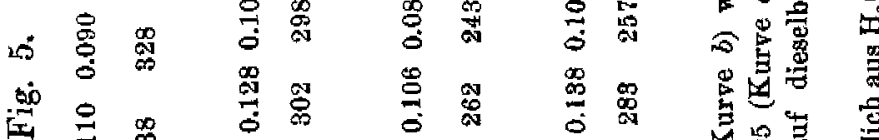

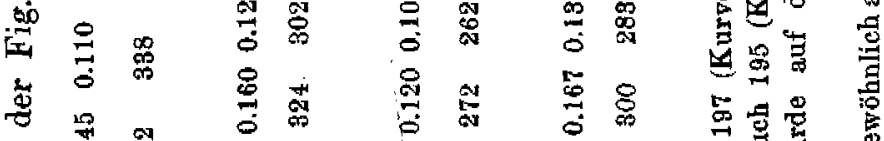

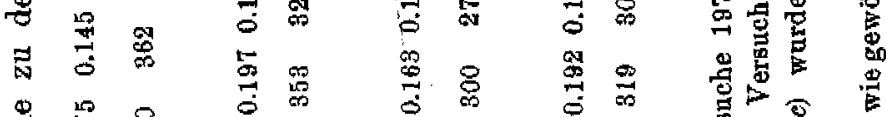

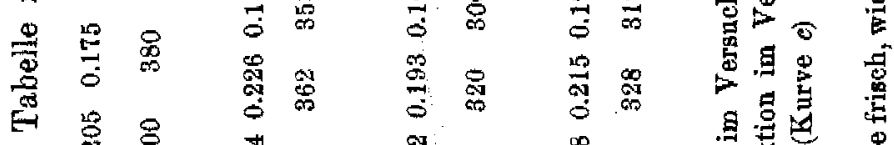

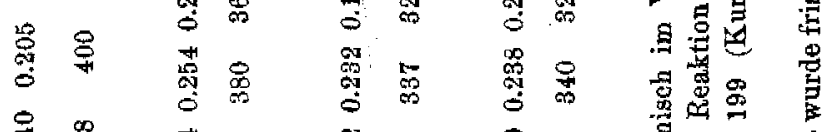

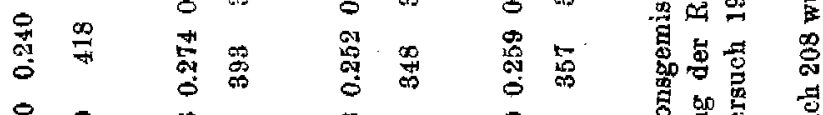

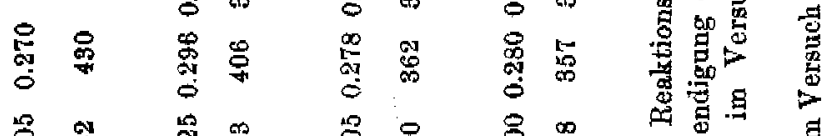

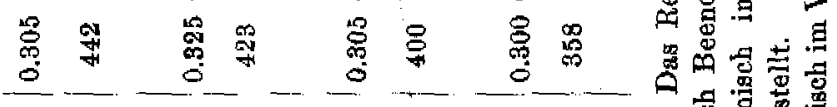

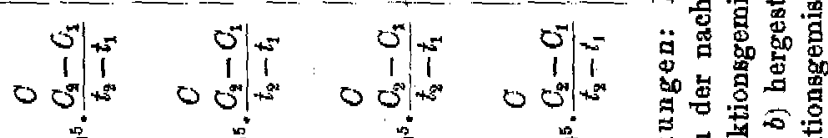

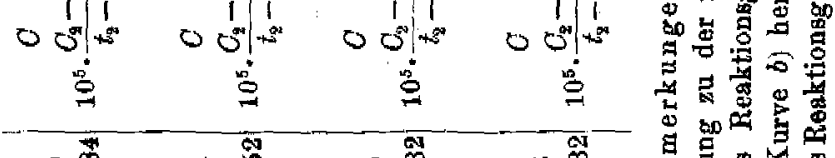

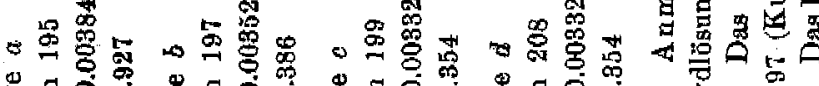

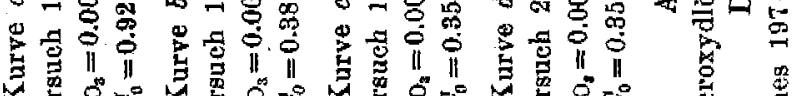

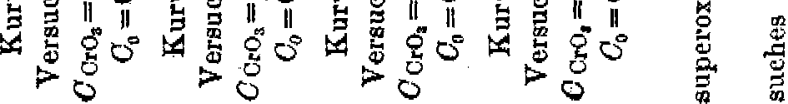


suchen $c$ und $d$ die gleichen. Die Kurven fallen vollständig zusammen. Daraus folgt, dafs in der bei den Versuchen $a, b$ und $c$ nacheinander angewandten Chromsäure, aufser der gleich am Anfang des er'sten Versuches stattgefundenen teilweisen Reduktion, keine weiteren irreversiblen Änderungen vorgekommen sind, während diese kleine Chromsäuremenge (0.000084 Mol.) im ganzen $0.039 \mathrm{Mol}$. reines Wasserst offsuperoxyd mit Entwickelung von $470 \mathrm{ccm} \mathrm{O}_{2}$ zersetzt und zweimal den heftigen Anstieg der Geschwindigkeit verursacht hatte. 'Diese kleine Menge kann folglich auch weiter unbegrenzte Mengen des Wasserstoffsuperoxyds mit demselben Reaktionsverlaufe zersetzen, ohne selbst dabei weiter zerstört zu werden. Daraus folgt, dafs der Zustand des Katalysators in allen angefuhrten Versuchen nicht nur im nahezu horizontalen Teile der 'Kurven, sondern auch in jedem beliebigen Punkte der gleiche bleibt, oder aber, wenn sich dieser Zustand mit fortschreitender Reaktion überhaupt ändert, so müssen diese Änderungen reversibler Natur mit einem sich momentan einstellenden Gleichgewicht sein, so dafs bei gegebener Temperatur der Zustand des ganzen, aus Wasserstoffsuperoxyd, zum Teil reduzierter Chromsäure und Wasser bestehenden, den Sauerstoff entwickelnden Systems durch dile jeweills vorhandene Wasserstoffouperoxydkonzentration von Anfang bis zum Ende der Reaktion vollständig definiert is t.

\section{Variable Chromsäurekonzentration.}

Es haben sich auch bei der kinetischen Untersuchung variabler Chromsäurekonzentrationen leicht übersichtliche Verhältnisse ergeben. Da, wie gezeigt, die Anfangskonzentration des Wasserstoffsuperoxyds ohne Einflufs auf den Reaktionsverlauf ist, so können die Versuche auch mit verschiedenen Anfangskonzentrationen des letzteren zum Vergleich mit herangezogen werden. Fig. 6 enthält die dies* bezbiglichen Resultate.

Im gradlinigen Teile verlaufen alle Kurven parallel und die Geschwindigkeiten, verglichen bei gleichen Punkten der Abszisse, sind ungefähr proportional der Anfangskonzentration der Chromsäure mit einer allerdings nicht grofsen Abweichung zugunsten der konzentrierten Chromsäurelösungen (s. Tabelle 3, fünfte und siebente Spalte). Das Ansteigen der Geschwindigkeit und ihr Maximum findet um so früher, d. h. bei um so gröfserer noch vorhandenen Konzentration des Wasserstoffsuperoxyds statt, je konzen. 
trierter die Chromsaure ist. Allerdings reicht die Genauigkeit der Mefsmethode doch nicht aus, um die Punkte der Abszisse, wo

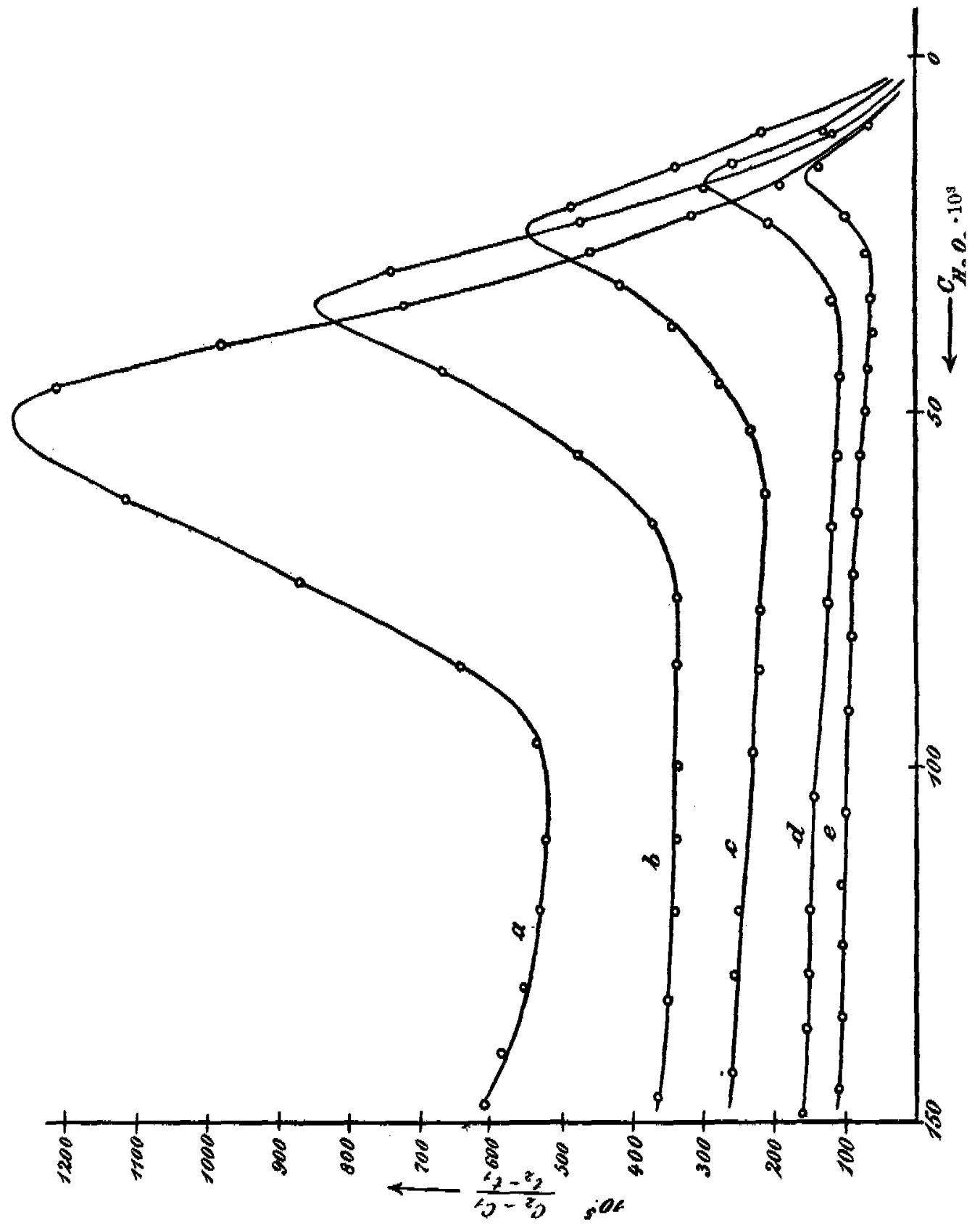


Tabelle 3 (vergl. Fig. 6).

\begin{tabular}{|c|c|c|c|c|c|c|c|c|c|}
\hline 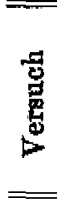 & 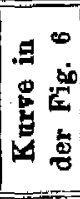 & 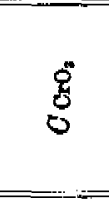 & 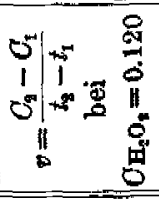 & $=\mid \int_{0}^{0}$ & 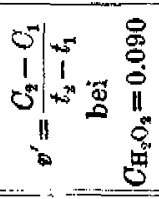 & 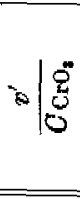 & $d$ & 量 & 㫷莺 \\
\hline 144 & $a$ & 0.00510 & 0.00520 & 1.0 & & & 0.050 & 10 & 0.11 \\
\hline 137 & $b$ & 0.00884 & 0.00850 & 0.9 & 0.00330 & 0.85 & 0.085 & 9 & 0.095 \\
\hline 119 & $o$ & 0.00298 & 0.00260 & 0.9 & 0.00220 & 0.7 & 0.025 & 8 & 0.08 \\
\hline 181 & $d$ & 0.00192 & 0.00150 & 0,8 & 0.00125 & 0.7 & 0.017 & 9 & 0.04 \\
\hline 111 & $e$ & 0.00149 & & & 0.00090 & 0.6 & 0.015 & 10 & 0,03 \\
\hline
\end{tabular}

das Maximum und noch weniger, wo das Minimum der Geschwindigkeit liegt, genugend genau zu bestimmen. Es ergibt sich trotzdem ziemlich deutlich die Proportionalität $z$ wischen der ursprunglichen Chromsäurekonzentration und der dem Maximumder Gesch windigkeit entsprechenden $\mathrm{H}_{2} \mathrm{O}_{2}$-Konzentration $C_{\max }$ (Spalte 8 und 9, Tabelle 3).

Durch graphische Interpolation lafst sich zeigen, dals auch die Geschwindigkeit selbst, welche bei jeder Chromsäurekonzentration im Maximum erreicht wird, ungefähr proportional der letzteren ist.

Wenn auọ mit gewisser Vorsicht, kann man aus diesen Resultaten schliefsen, dafs bei konstanter Temperatur die Geschwindigkeit der Wasserstoffsuperoxydzersetzung auch bei verschiedenen Chromsäurekonzentrationen einerseits durch diese, andererseits durch die in jedem Momente vorhandene Wasserstoffsuperoxydkonzentration eindeatig bestimmt wird.

\section{Übergang zu der monomoleknlaren Reaktion.}

Obwohl der Unterschied im zeitlichen Verlaufe der katalytischen Wirkung freier Dichromsäure und derjenigen ihres Neutralsalzes, des Dichromates, sehr grofs ist, lärst sich ein Übergang zwischen beiden doch leicht experimentell realisieren. Der Hauptgrund des Unterschiedes liegt in erster Linie darin, dals, während von der freien Chromsäure in den von mir untersuchten Versuchsbedingungen etwa $28 \%$ reduziert werden, das Dichromat am Ende der Wasserstoffsuperoxydkatalyse sich als intakt geblieben erweist. ${ }^{1}$ Diese Reduktion für sich allein erklärt aber noch bei weitem nicht den grolsen Unterschied in der Kinetik zwischen der Reaktion erster Ordnung ${ }^{2}$

1 Vergl. Erste Mitteilung, I. c. 8. 188, Tabelle 1.

Daselbst S. 186. 
und der oben wohl überhaupt zum erstenmal beschriebenen Art der Katalyse. ${ }^{1}$

Um den Übergang zwischen diesen beiden kinetischen Typen zu realisieren, braucht man nur in der Dichromsäure das $\mathrm{H}^{-}$-Ion nach und nach durch das Kaliumion zo ersetzen, entweder durch allmähliche Neutralisation derselben mit Kalilauge, oder, was dasselbe ist, durch entsprechendes Zusammenmischen freier Chromsäure mit Kaliummonochromat. Fig. 7 stellt ein Beispiel eines solchen, wenn auch nicht ganz allmählichen, Überganges dar.

Man sieht zunächst, dafs die Geschwindigkeit im ersten Teile der Reaktion bei freier Chromsäure (Kurve a) eine viel kleinere als bei der äquivalenten Menge Dichromat (Kurve $c$ ) ist, wobei dieser Unterschied, (welcher zuerst viel gröfser als der dem reduzierten Bruchteile der freien Chromsäure entsprechende ist, ${ }^{2}$ ) mit fortschreitender Reaktion immer kleiner wird, bis sich die Kurven etwas über dem Minimum bei freier Chromsäure schneiden, um dann wieder weit auseinander zu gehen, so dafs die dem Maximum entsprechende Geschwindigkeit bei der Dichromsäure $8 \mathrm{mal}$ so grols ist, als die Geschwindigkeit bei Dichromatkatalysator und derselben $\mathrm{H}_{2} \mathrm{O}_{2}$ - Konzentration ( 0.03 Mol. pro Liter). Ersetzt man das $\mathrm{H}^{*}$-Ion freier Dichromsäure durch Kaliumion (Versuch $b$ ) nur soweit, dafs der Katalysator ein Gemisch aus äquivalenten Mengen Dichromsäure und Kaliumdichromats darstellt, dann zeigt sich die folgende merkwürdige Erscheinung: Die Anfangsgeschwindigkeit (Kurve b) steigt sogar über die des reinen Kaliumdichromats (Kurve o) hinauf, nimmt jedoch mit abnehmender Wasserstoffsuperoxydkonzentration viel stärker, als bei reiner Dichromsăure (Kurve a) ab und geht zunächst beinahe parallel derjenigen des Dichromats, um sich dann allmählich von dieser zu entfernen und darauf einen ebenso grofsen und scharfen Anstieg, wie bei reiner Chromsähure (Kurve a) durchzumachen. Weitere Versuche mit derselben konstanten Gesamtchromkonzentration wurden nicht gemacht, es lälst sich jedoch aus anderen weiter unten mitzuteilenden Versuchen sehen,

1 Die weitere Unfersuchung wird eventuell zeigen, in welchen Zusammenbang mit der gebräuchlichsten Theorie dor Katalyse, d. h. der Theorie der Folgereaktionen, der hier beschriebene Fall zu bringen wåre.

- Bei steigender Wasserstoffsuperoxydkonzentration wird dieser Unterschied inmer gröfser, weil die Geschwindigkeit bei Dichromat proportional der Superoxydkonzentration (Reaktion orster Ordnung), dagegen bei freier Chromsäure nur ungefähr proportional der dritten Wurzel aus derselben ist. 
dals, je mehr sich die ursprüngliche Zusammensetzung des Katalysators derjenigen des Dichromats nähert, um so deutlicher und genauer der erste Teil der Kurve derjenigen des Dichromsts parallel

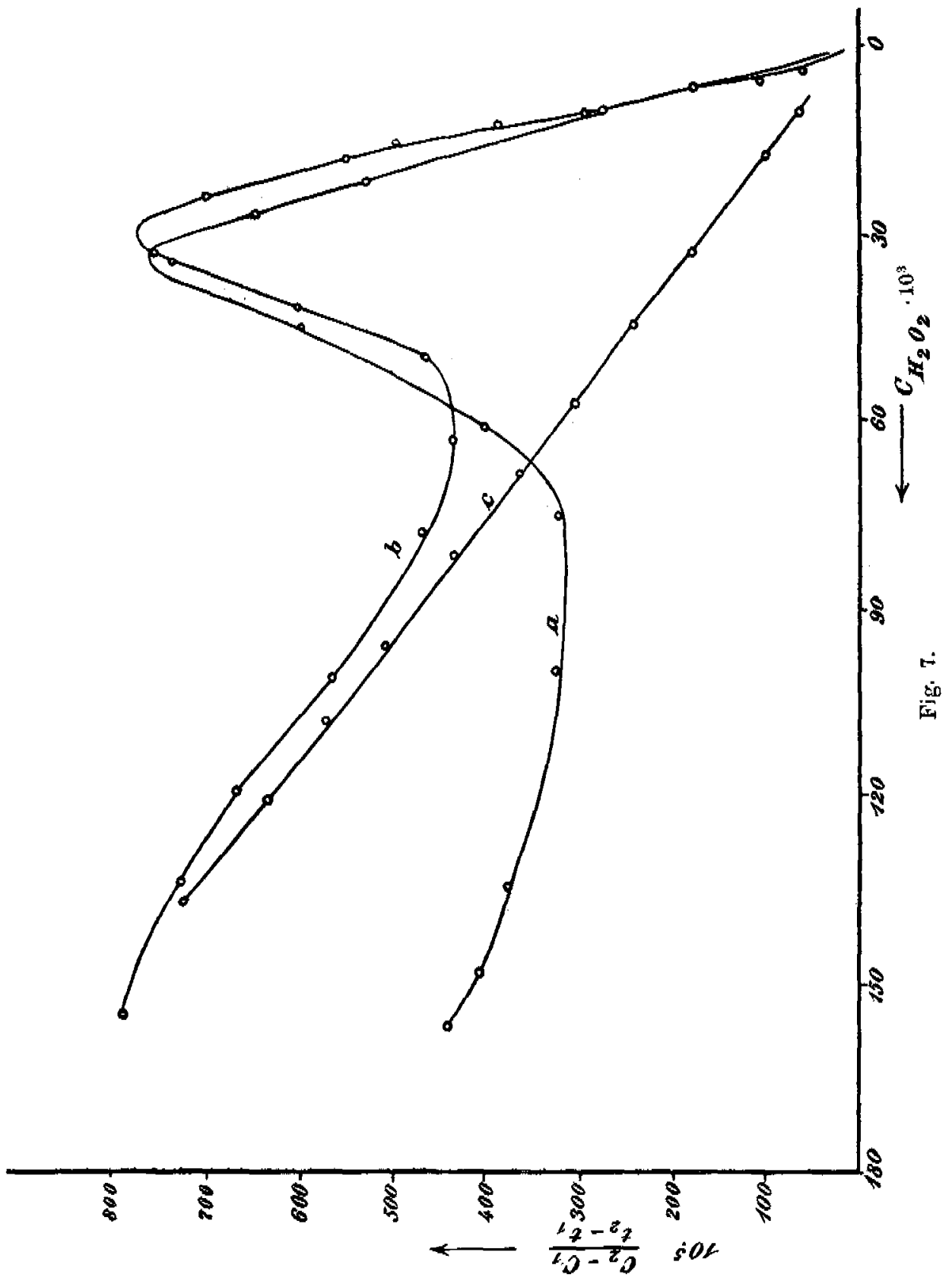




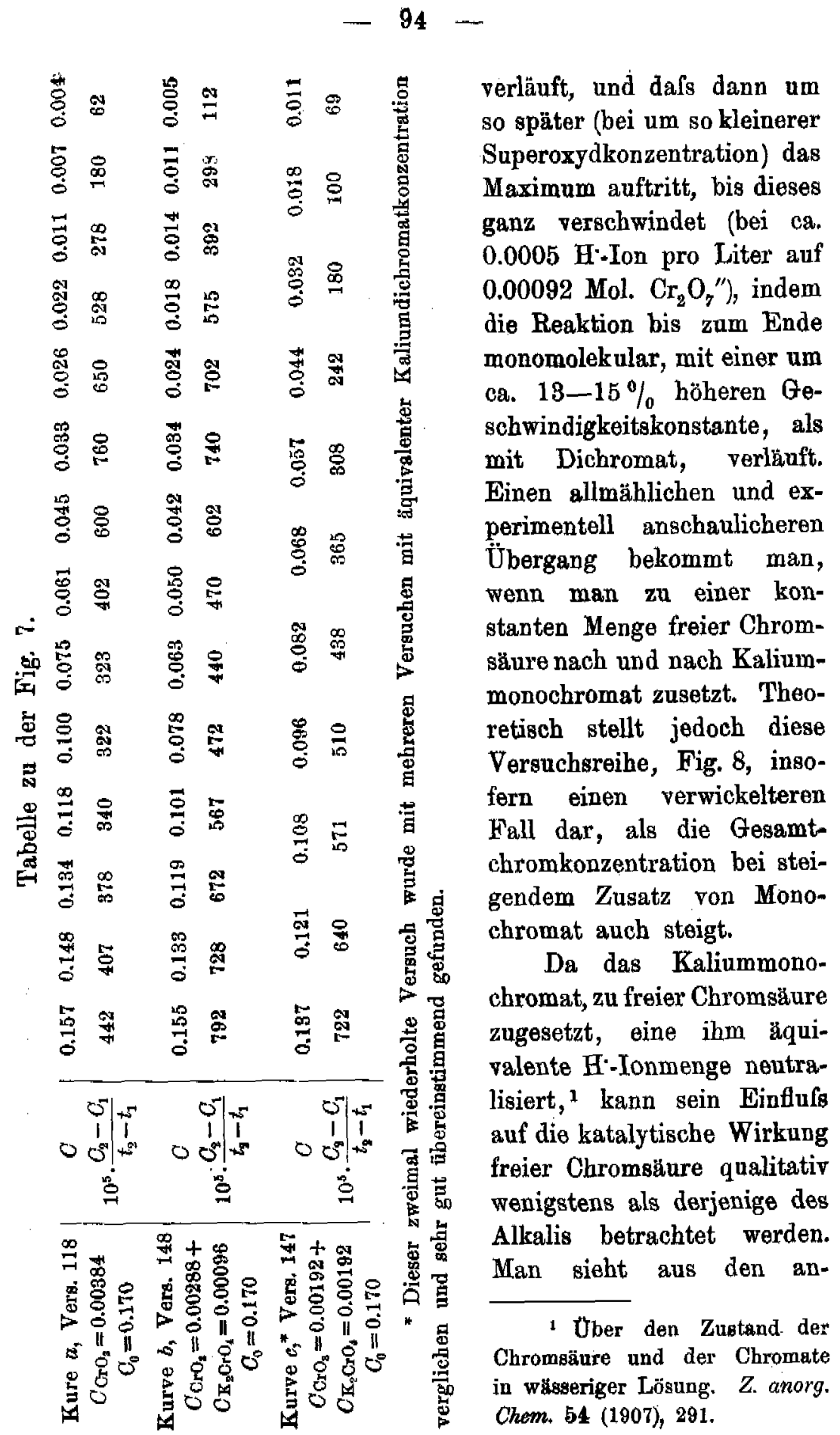


gefürten Beispielen (Fig. 8 und besonders Fig. 7), welch grofsen Finflufs auf das Zeitgesetz des Reaktionsverlaufes die An- bzw. Abwesenheit des H'-Iones ausubt.

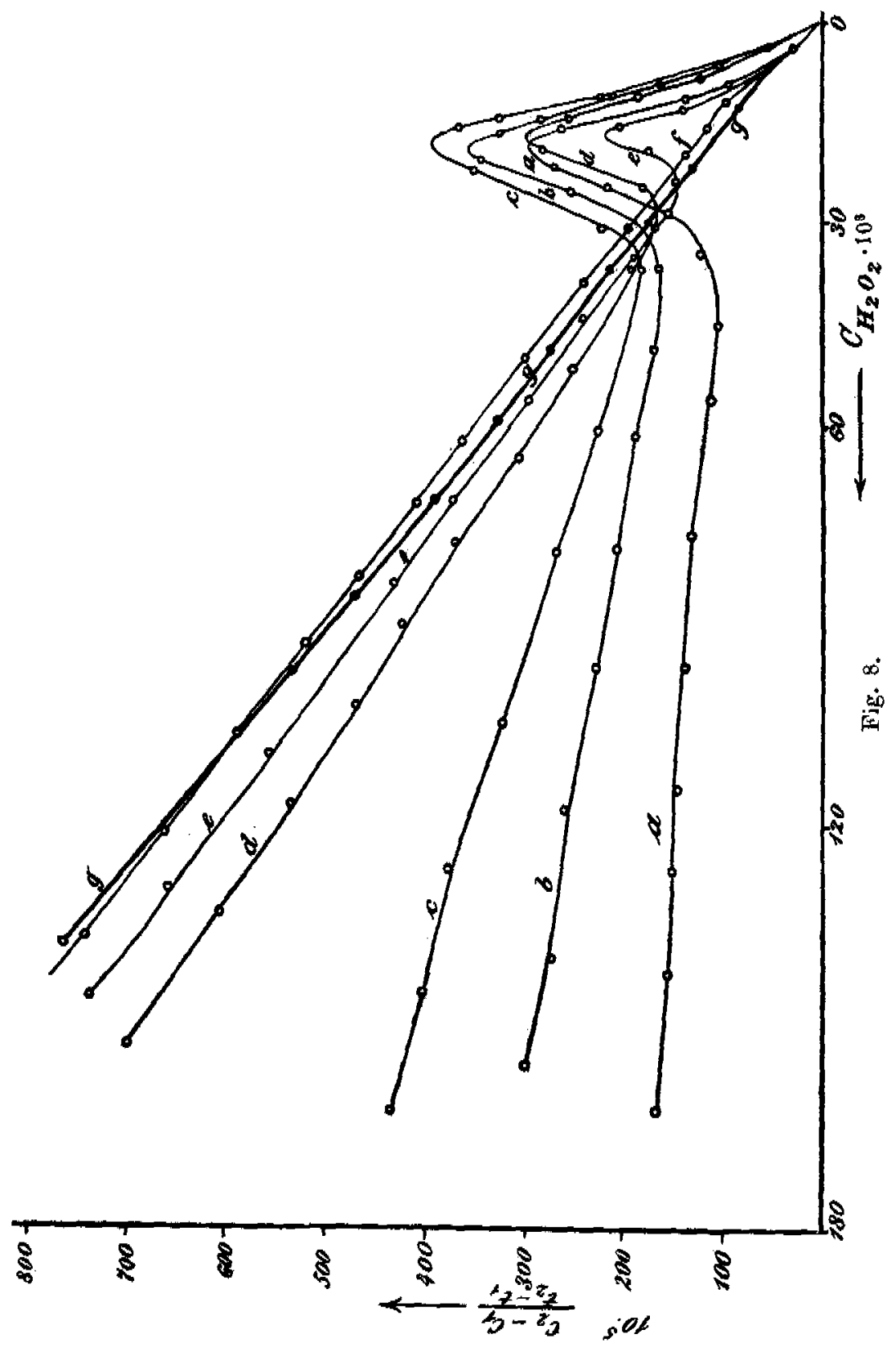


Tabelle zu der Fig. 8.

\begin{tabular}{l|l|l|l}
\hline Versuch & Kurve & $O_{\mathrm{CrO}_{\mathrm{g}}}$ & $O_{\mathrm{K}_{2} \mathrm{CrO}_{4}}$ \\
\hline 181 & $a$ & 0.00192 & 0 \\
136 & $b$ & 0.00192 & 0.000228 \\
126 & 0 & 0.00192 & 0.000456 \\
127 & $a$ & 0.00192 & 0.000912 \\
143 & $e$ & 0.00192 & 0.00124 \\
130 & $f$ & 0.00192 & 0.00156 \\
147 & $g$ & 0.00192 & 0.00192
\end{tabular}

Dieser Einflufs ist, wie schon frtiher betont, viel gröfser und komplizierter, als wie er nur durch die teilweise Reduktion der Chromsäure zustande käme. Man kann schlechthin sagen, dafs das Wasserstoffion hier auch katalytisch mit einer durchgreifenden Änderung der „Reaktionsordnung" wirkt, nur sagt, eine solche rein formale Definition nichts weiter aus, bis eine nähere Untersuchung den Mechanismus der Reaktion aufklärt.

Um die Möglichkeit irgend eines experimentellen Mifsverständnisses am Anfang der vorliegenden Untersuchung auszuschliefsen, habe ich mich aufser der Variation der benutzten Präparate, bauptsächlich solcher Versuchsreihen bedient, welche den Übergang zwischen der mitgeteilten Art der Katalyse und der monomolekularen Reaktion (Fig. 7 und 8) und umgekehrt realisierten. Vielleicht wird es nicht überflüssig sein, wenn ich hier noch eine solche Versuchsreihe anführe, bei welcher zu einer Kaliumdichromatlösung sehr verdünnte Salpetersäure zugesetzt wurde (Fig. 9, S. 97).

Tabelle zu der Fig. 9.

\begin{tabular}{c|c|c|c}
\hline Verauch & Kurve & $C_{\mathrm{K}_{2} \mathrm{Cr}_{8} \mathrm{O}_{2}}$ & $O_{\mathrm{HNO}}$ \\
\hline 147 & $a$ & 0.00192 & 0 \\
184 & $b$ & 0.00192 & 0.000405 \\
183 & $e$ & 0.00192 & 0.00101 \\
178 & $a$ & 0.00192 & 0.00251 \\
177 & $e$ & 0.00192 & 0.00503 \\
182 & $f$ & 0.00192 & 0.00761 \\
181 & $g$ & 0.00192 & 0.0152
\end{tabular}

Während ein Zusatz von 0.000405 Mol. Salpetersäure auf 0.00192 Mol. Dichromat noch keine merkliche Änderung in der Form des Reaktionsverlaufes mit Wasserstoffsuperoxyd hervorruft 
(Kurve b), findet schon bei 0.001 Mol. Salpetersäure ein kleines Aufsteigen der Geschwindigkeit an Einde der Reaktion statt (Kurve $c$ ). Andererseits geht die Katalyse schon bei einem Zusatz von $0.0076 \mathrm{Mol}$.

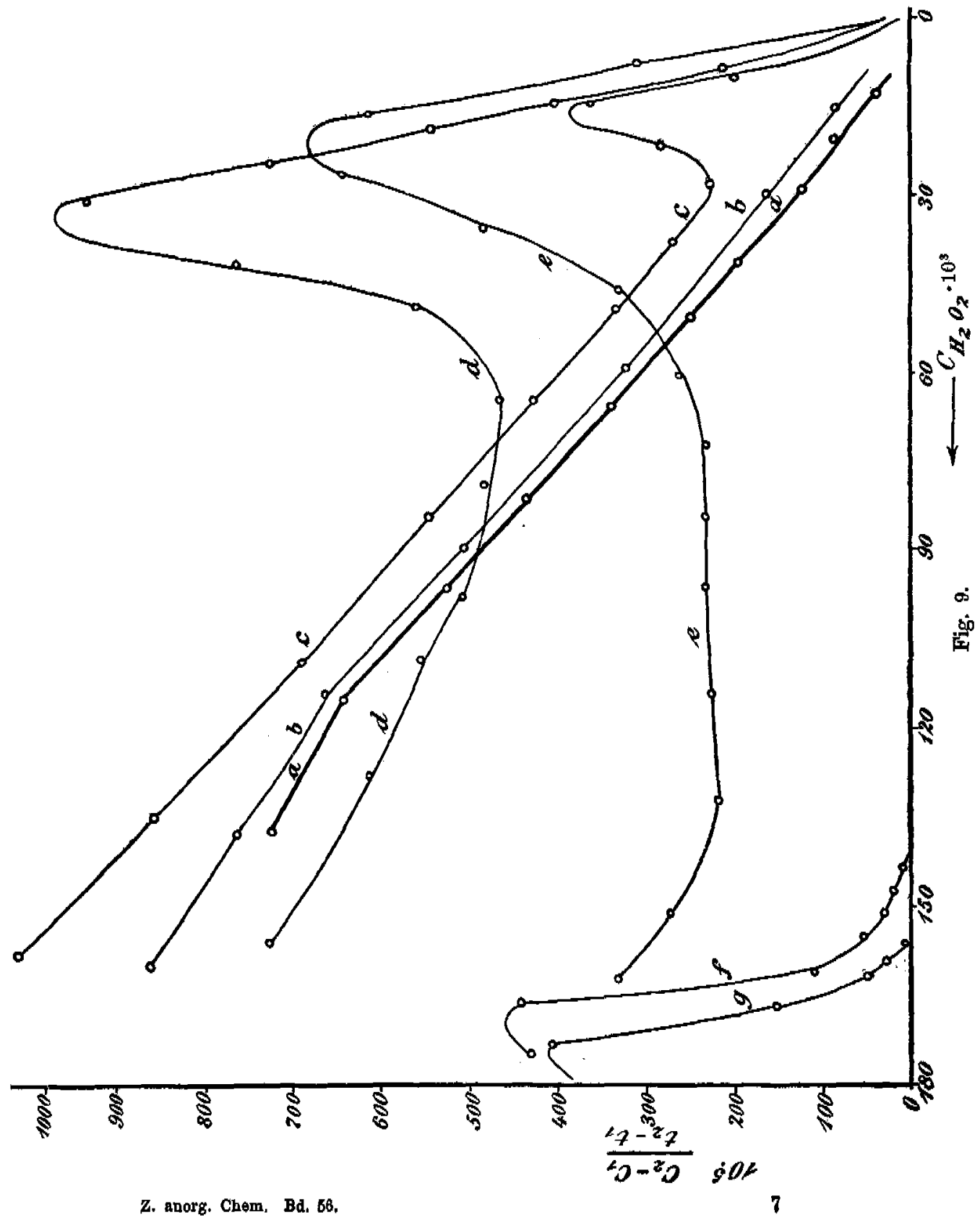


$\mathrm{HNO}_{3}$ auf $0.0019 \mathrm{~K}_{2} \mathrm{Cr}_{2} \mathrm{O}_{7}$ sehr bald fast unmefsbar langsam vor sich (Kurve f). Also zwischen 0.0004 und $0.007 \mathrm{Mol}$. Salpetersäure auf 0.00192 Mol. Dichromat liegt das Gebiet der ungemein grofsen Mannig-

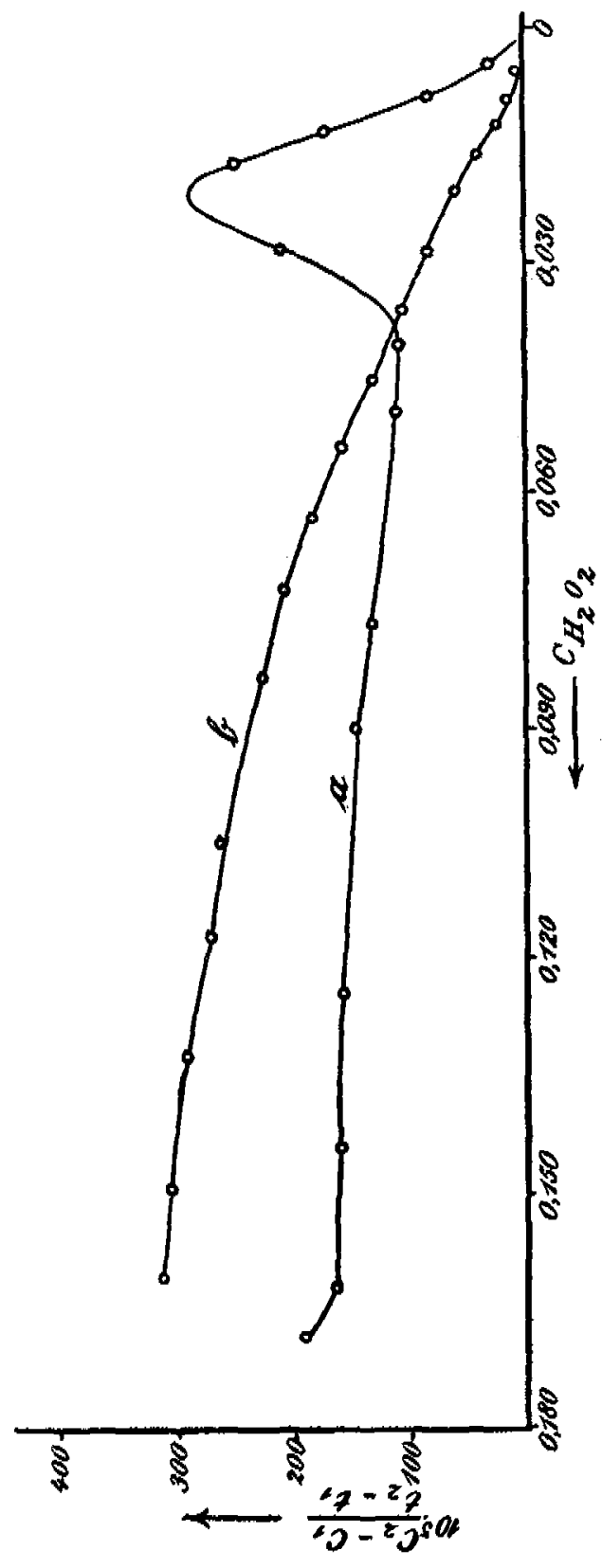

faltigkeit des Reaktionsverlaufes. Auch Zusatz von Natriumacetat $z u$ einer $\mathrm{CrO}_{3}$ - Auflösung kann den für die freie Chromsäure charakteristischen Reaktionsverlauf sehr stark verändern, wie das die Fig. 10 zeigt.

(S. Tabelle, S. 99.)

Das Aufsteigen der Reaktionsgeschwindigkeit am Ende der Reaktion ist in Gegenwart von Acetat verschwun-

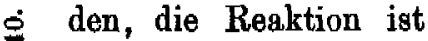
aber noch nicht rein monomolekular, indem die monomolekulare Geschwindigkeits k o n stante ein Ansteigen (bis etwa 25\%) aufweist, wie auch aus der Abweichung der Kurve $b$ (Fig.10) von der geraden Linie zu ersehen ist.

Einiges über den Reaktionsverlanf.

Aus allen oben mitgeteilten Resultaten haben wir geseben, wie mannigfaltig der Reaktionsverlauf in einem scheinbar noch so ein- 


\begin{tabular}{c|c|c|c}
\hline Verguch & Kurve & $C_{\mathrm{CrO}_{3}}$ & $C_{\mathrm{NaC}_{2} \mathrm{H}_{3} \mathrm{O}_{2}}$ \\
\hline \hline 185 & $a$ & 0.00205 & 0 \\
192 & $b$ & 0.00205 & 0.00453
\end{tabular}

fachen Reaktionssysteme (Chromsäure - Wasserstoffsuperoxyd) sein kann, und wie leicht sich die scheinbar so verschiedenen Arten der Katalyse ineinander verwandeln. Eine solche Mannigfaltigkeit würde an und für sich auf eine ungewöhnliche Kompliziertheit der während und bei der Katalyse sich gleichzeitig abspielenden chemischen Vorgänge hindeuten und dadurch die nähere Untersuchung des Reaktionsmechanismus mit unseren doch noch so unvollkommenen Mitteln hoffnungslos machen, wenn sich durch meine Versuche nicht eine Erscheinung herausgestellt hätte, welche allen oben mitgeteilten Tatsachen zugrunde zu liegen scheint. Das ist die oben (S. 89 n. 91) gezeigte Unabhängigkeit der Reaktionsgeschwindigkeit bei jeder noch vorhandenen Wasserstoffsuperoxydkonzentration von der "Vorgeschichte“ der Reaktion, d. h. davon, wie lange schon die gegebene Chromsäuremenge mit $\mathrm{H}_{3} \mathrm{O}_{3}$-Lösung reagiert hat. Diese Erscheinung, wie schon oben gesagt, dentet darauf hin, dafs die während der Wasserstoffsuperoxydzersetzung stattfindenden Änderungen in dem Zustande des Katalysators (seine Reduktion zum Beispiel) nicht als mit dex Katalyse parallel gehende unabhängige Nebenreaktionen aufzufassen sind, sondern dals die eventuellen Änderungen des alsbald nach dem Zusammenmischen der Lösungen von Wasserstoff. superoxyd und Chromsäure angenommenen Zustandes des Katalysators solche reversibler Natur sind. Dieser Zustand (in bezug auf die Geschwindigkeit der $\mathrm{H}_{2} \mathrm{O}_{2}$-Zersetzung) ist bei gegebener Anfangskonzentration des Katalysators in jedem Augenblicke nur durch die vorhandene Wasserstoffsuperoxydkonzentration bestimmt. Es ist natürlich klar, dafs ich hier nicht etwa eine echte Beversibilitut der katalytischen $\mathrm{H}_{2} \mathrm{O}_{2}$-Zersetzung meine, was schon durch die irreversible Sauerstoffentwickelung ausgeschlossen wäre; ich glaube vielmehr ein, wenn auch ziemlich kompliziertes, Oxydations-Reduktionsgleichgewicht der Chromsäure vor mir zu haben, welches durch die Unbeständigkeit der höheren Oxydationsprodulte der Chromsäure fortwăhrend gestört und durch die dadurch zustande kommende Zersetzung des Wasserstoffsuperoxydes und Abnahme seiner Konzentration immerwăbrend verschoben wird. 
Ich bin noch nicht in der" Lage, über die Natur dieses Gleichgewichtes etwas näheres und experimentell begründetes mitzuteilen, ebenso wie ich noch nicht behaupten möchte, dafs diese Interpretierung meiner Beobrchtungen die einzig mögliche ist, - ein grofser Betrag an Wahrscheinlichkeit kommt ihr jedoch zu.

Trotz vieler Bemühungen ist mir bis jetzt leider nicht gelungen, eine genaue Methode auszuarbeiten, um zu untersuchen, ob und wie sich das Verhältnis zwischen sechs- und dreiwertigem Chrom in Gegenwart von Wasserstoffsuperoxyd mit fortschreitender Katalyse ändert, weil die Kleinheit der hier in Betracht kommenden Mengen und die Anwesenheit grofser Mengen Wasserstoffsuperoxyd diese Bestimmungen sehr erschweren, besonders wenn man bedenkt, dafs der Zusatz eines jeden sauer resp. alkalisch reagierenden -Stoffes in Gegenwart von Wasserstoffsuperoxyd das zu bestimmende Verhältnis eventuell momentan verschieben kann. Die Methoden der Farben- resp. Leitfähigkeitsmessung dürten hier wohl noch am weitesten führen. Ich habe jedoch, wenn auch noch nicht ganz einwandfreie Andeutungen dafïr, dal's sich das nach der vollständigen Zersetzung des. Wasserstoffsuperoxyds zurückbleibende Verhältnis zwischen unreduzierter und reduzierter Chromsäure auch von der anderen Seite her erreichen lärst. Man bekommt nämlich dasselbe Verhaltnis $\frac{72 \mathrm{Cr}^{\mathrm{7I}}}{28 \mathrm{Cr}^{111}}$ (Tabelle 1, Seite 80), wenn man von einer Lösung ausgeht, welche schon etwa $40 \%$ ihrer Chromkonzentration in Form von dreiwertigen Chrom enthält und wenn man auf diese Lösung einen Überschufs vou Wasserstoffsuperoxyd einwirken läfst, nur darf die Lösung keine fremden Kationen (aufser $\mathrm{H} \cdot$-Ion) resp. Anionen enthalten, damit man das richtige Verhältnis bekommt. ${ }^{1}$

Es ist bis jetzt auch nicht entschieden worden, welche von den Überchromsüuren als Zwischenprodukt bei der Katalyse mit freier Chromsüure ebenso wie mit ihren Salzen entsteht. Der Farbe nach soll das eine und dieselbe sein.

Es ist auch nicht ausgeschlossen, dal's in Gegenwart von $\mathrm{H}^{-}$-Ion und verschiedenen Wasserstoffsuperoxydkonzentrationen sich verschiedene, verschieden schnell zerfallende Überchromsäuren bilden, und $\mathrm{zwar}$ bei abnehmender $\mathrm{H}_{2} \mathrm{O}_{2}-$ Konzentration immer mehr von der schneller zerfallenden, was das Auftreten des Minimums und

${ }^{1}$ Die Untersuchung dicser interessanten Verhaltwisse wird fortgesetzt. 
Maximums am Ende der Reaktion vielleicht erklären könnte. Woch möchte ich ron der Aufstellung solcher vor der Hand rein spekulativen Hypothesen bis zur Auffindung weiterer experimenteller Anhaltspunkte absehen, ebenso wie von der Aufsuchung einer rein empirischen Formel, welche den oben beschriebenen Reaktionsverlauf wiedergäbe. ${ }^{1}$

Eines steht bis jetzt fest, dals die Geschwindigkeit in jedem Augenblicke durch die anwesende Wasserstoffsuperoxydkonzentration eindeutig bestimmt wird und dafs diese Geschwindigkeit ceteris paribus sehr empindlich gegen $\mathrm{H}^{\circ}$ bzw. $\mathrm{OH}^{\prime}$ ist.

Als eine hübsche Illustration dieser Empfindlichkeit möchte ich noch zum Schlufs erwähnen, dafs man die Katalyse des. Wasserstoffsuperoxyds durch Chromate als Indikator in der Acidimetrie verwenden kann, abgesehen natürlich von der praktischen Bedeutung einer solchen Anwendung. Von SснӧNBEIN ${ }^{2}$ wurde schon beobachtet, dafs die lasurblaue Farbe eines schwachsauren Gemisches von Überchromsäure und Wasserstoffsuperoxyd darch einen Tropfen Alkali in die violette (rotbraune?) tibergeht und umgekehrt, welche Erscheinung RrEsENEELD ${ }^{3}$ durch die Schwäche der Überchromsäuren erklärt hat.

Viel schärfer und theoretisch interessanter als dieser den gewöhnlichen Indikatoren analoge Umschlag ist aber folgender: Wie

1 Die einfachste von mir abgeleitete Differentialgleichung:

$$
-\frac{d c}{d i}=k\left(\frac{c^{8}}{6}-\frac{n c^{2}}{2}+m\left(n-\frac{m}{2}\right) c\right)
$$

welche die Bedingungen erfüllt, dals die Reaktionsgeschwindigkeit $-\frac{d c}{d t}$ bei gegebener Chromaăurekonzentration in eindeutig bestimmten Punkten der Abszigse (noch vorbandene Wasserstoffsuperoxydkonzentration $C_{\mathrm{H}_{2} \mathrm{O}_{2}}$ ) durch ein Minimum, einen Wendepunkt $\left(C_{\mathrm{H}_{2} \mathrm{O}_{2}}=n\right)$ und durch ein Maximum $\left(C \mathrm{H}_{8} \mathrm{O}_{2}=m\right)$ geht, hat sich nicht als brauchbar zur Darstellung der Versuchsergebnissen gezeigt, was patürlich darauf hindeutet, dafs die dabeigemachte Voraussetzung $\frac{d^{2}(-d o / d t)}{d c^{2}}=k(o-n)$ nicht die genügende ist, um den tatä̈chlichen Verlauf der Kurve (Reaktionsgeschwindigkeit als Funktion von $C_{\mathrm{F}_{2} \mathrm{O}_{\mathrm{p}}}$ ) wiederzugeben. Jede andere Beziehung $\frac{d^{2}(-d e / d t)}{d c^{2}}=k \cdot F(c-n) F(c)$ würde auch dieBedingung $\frac{d^{2}(-d c / d t)}{d c^{2}}=0$, wenn $o=n$, erfitlen.

¿ Journ. prakt. Chem. $80(1860), 257$.

8 Zur Kenntnia der Überchromsäuren und ihrer Salze, Habilitationsschrift, Freiburg 1906, S. 44. 
ich in der ersten Mitteilung gezeigt habe, findet eine katalytische Zersetzung des Superoxyds bei verdünnten Kaliummonochromatlösungen praktisch nicht statt und die hellgelbe Farbe des Mono. chromats bleibt beim $\mathrm{Zusatz}$ von viel $\mathrm{H}_{2} \mathrm{O}_{2}$ unverändert. Gielst man nun etwa $5 \mathrm{ccm}$ eines solchen gelben Gemisches aus wenig Kaliummonochromat und relativ viel $\mathrm{H}_{2} \mathrm{O}_{2}$ in eine abgemessene Säurelösung (z. B. Salpetersäure), dann wird das Monochromat momentan reduziert, die Fltussigkeit ist grin, und es geht wieder keine Katalyse vor sich. Lallst man jetzt Alkali eintropfen, dann wird nach einer bestimmten Menge des Alkalizusatzes die Flüssigkeit, welche vorher trotz des schönen Farbenspieles an der Eintropfstelle beim Durchmischen noch immer grün blieb, auf einmal (aber noch nicht genuggend scharf) rotbraun und die Katalyse setzt ein. Bei weiterem Eintropfen der Alkalilösung wird die rotbraune Farbe intensiver, die Katalyse energischer und dann färbt sich die Lösung plötzlich durch einen nächsten Tropfen Alkali hellgelb, wobei sofort die $\mathrm{H}_{2} \mathrm{O}_{2}$-Zersetzung aufhört. Bei wiederholten Versuchen zeigt sich die dabei im ganzen verbrauchte Alkalimenge bis $0.5 \%$ genau konstant und gleich derjenigen, welche verbraucht wird, wenn man dieselbe Menge Säure mit derselben Alkalilösung und Phenolphtalein als Indikator titriert (Tabelle 4).

Tabelle 4.

\begin{tabular}{|c|c|c|}
\hline $\begin{array}{c}\text { Genommene Salpeterszure } \\
0.1 \text { norm. in ccm }\end{array}$ & $\begin{array}{l}\text { Verbrauchte Natronlauge } \\
\text { in ccm }\end{array}$ & Indikator \\
\hline$==-=$ & $===$ & $======$ \\
\hline 20 & 18.3 & \multirow{3}{*}{$\begin{array}{c}\text { Katalyse d. Wasser- } \\
\text { stoffsuperoxyds } \\
\text { duxeb Chromate }\end{array}$} \\
\hline 20 & 18.3 & \\
\hline 30 & 27.5 & \\
\hline 20 & 18.3 & \multirow{2}{*}{ \} Phenolphtalein } \\
\hline 20 & 18.3 & \\
\hline
\end{tabular}

Von dem katalytisch unwirksamen Monochromat ausgehend, kommt man durch den anderen katalytisch inaktiven Zustand (saure Lösung) und durch das schmale Gebiet starker Katalyse zu derselben hellgelben nichtreagierenden Monochromatlösung, und hierzu ist notwendig, dafs die genommene Säure genau neutralisiert wird. 


\section{Sohlufs.}

Die weitere Untersuchung des Mechanismus der oben beschriebenen Reaktion soll meine nächste Aufgabe sein. ${ }^{1}$

Indessen gibt mir diese Reaktion schon jetzt die Veranlassung, auf ihre allgemeinere Bedeutung als Katalyse mit sichtlicher Zwischenverbindung zwischen Substrat und Katalysator, insbesondere bezüglich ihrer wenn aùch vor der Hand rein formalen, Analogie mit sogenannten Fermentreaktionen hinzuweisen.

Die Fälle solcher mehr oder weniger ausführlich kinetisch untersuchter Fermentreaktionen werden immer zablreicher und immer deutlicher treten die kinetischen Regelmälsigkeiten bei diesen Reaktionen hervor.

Die kinetische Eigentümlichkeit ${ }^{2}$ der meisten Fermentreaktionen besteht darin, dass, während bei einem Überschusse von Ferment die Reaktion mehr oder weniger genau das monomolekulare Zeitgesetz ergibt, die Reaktionsgeschwindigkeit bei einem Überschusse von Substrat weniger mit dem Fortschreiten der Reaktion abnimmt, als proportional der Substratkonzentration d. b. als einer monomolekularen Reaktion entsprechend, und dafs sie in manehen Fallea sogar scheinbar unabhängig von der vorhandenen Substratmenge ist.

Diese meistens nur angenäherten Regelmälsigkeiten werden von verschiedenen Autoren in Form ron einzelnen, flur die Fermentreaktionen angeblich "charakteristischen" Sätzen zum Ausdruck gebracht, so z. B: Wenn die Substratmenge ein Maximum abersteigt, dann ist die pro Zeiteinheit umgewandelte Menge, die Geschwindigkeit, nur der Enzymkonzentration proportional (Duclaux, Tammane, O'Sulurvan und Tompson, Armstrong, Henri, Terroine u. a. ${ }^{3}$ ).

Bei gleicher Anfangsmenge des Substrats nähert sich mit abnehonder Fermentmenge die Kurve des Umsatzes (d. h. umgewandalte Substratmenge als Funktion der Zeit) immer

${ }^{1}$ Ich erlaube mir die Herren Fachgenossen darauf aufmerksam zu machen, dafs ich mir die ausführliche analoge Untersuchung der Wasserstoffsuperoxydkatalyse durch Molybdate, Wolframate, Vanadate u. a. ahnliche Verbindungen vorbehalten möchte.

2 Arrmexuog, Imamunochemie, Leipzig 1907; vergl. auch Euter, Zeitschr. physiol. Chem. 52 (1007), 147 u. w.

${ }^{3}$ Arragungs l. e., S. 38-40. 
mehr der geraden Linie. ${ }^{1}$ Dann erscheint also der Umsatz proportional der Zeit, die Geschwindigkeit unabhängig von der Zeit.

Bei gleichbleibenden Fermentmengen nimmt der Umsatz in Prozenten der Substratkonzentration ausgedrückt mit Erhöhung der Substratmenge ab. ${ }^{2}$

Wenn man auch in der letzten Zeit im Anschlufs an die Auffassung von Berzelius, Sciönbeiv und Ostwald und von Bredig $\$$ Untersuchungen iber „Anorganische Fermente" und dank Ducladux, Tammann, Arrennius u. a. die rein vitalistischen Theorien der Enzymwirkung fast allgemein verlassen hat, so werden doch noch jetzt die oben geschilderten kinetischen Eigentümlichkeiten der Fermentreaktionen für ein spezifisches Merkmal der Fermente gegenïber den anorganischen Katalysatoren gehalten, was seinen Grund vielleicht darin hat, dals solche eigentümliche Zeitgesetze, wie bei der Fermentwirkung, bei der anorganischen Katalyse, zumal im homogenen System, kaum bekannt sind. Zwar hatten BREDIe und MÜLLER voN BERNECK ${ }^{3}$ unter gewissen Umständen bei der ,mikroheterogenen" Platinkatalyse von alkalischem Wasserstoffsuperoxyd ebenfalls bereits eine der Zeit einfach proportionale Umsatzmenge gefunden, aber auch im homogenen System dürften sich solche Fälle bei der so grofsen kinetischen Mannigfaltigkeit theoretisch wenigstens wohl finden lassen. So kann z. B. schon eine gewöhnliche Katalyse mit Selbstbeschleunigung unter Umständen die Möglichkeit eines solchen Reaktionsverlaufes, wie bei Fermenten, ergeben. ${ }^{4}$ Auch einer der einfachsten Spezialfälle der Folgereaktionen enthält die Unabhängigkeit der Reaktionsgeschwindigkeit von der Konzentration des Substrats, solange dasselbe im Überschusse ist, als eine Notwendigkeit. ${ }^{5}$

1 Agderhalden und Michaetis, Zeitschr. physiol. Ohem. 52 (1907), 331.

2 O. Henzod, Zeitschr. physiol. Chem. 48 (1906), 366 ,

b Zeitsehr. phys. Chem. 31 (1899), 305.

- Wenn nämlich die jeweilige Zunghme der Gesehrvindigkeit infolge von Selbstbeschleunigung (Autokatalyse) gerade ibre durch Abnahme der Substratkonzentration entstehende Verlungsamung kompensiert.

${ }^{s}$ Wenn die erste aus zwei möglichen, nicht umkehrbaren Zwischenreak. tionen zwischen dem im grofsen Überschusse vorhandenen Substrat und dem Katalysator relativ sehr schnell verläuft und die zweite relativ sehr langsam, dann ist die Konzentration des Zwischenproduktes eine konstante und folglich auch die dann allein bestimmende Geschwindigkeit seiner Zersetzung. Vergl. Mrucor, Chemical statics and dynamics. London 1904, p. 375. Vergl. auch Mouron, Referat über den von Bredig und Bernecr beobachteten Reaktionsverlauf der $\mathrm{H}_{2} \mathrm{O}_{2}$-Zersetzung (siehe oben). Ann. de l'Institut Pasteur $14(1900), 573$. 
Ein Fall von konstanter Geschwindigkeit, anscheinend unabhängig von der Substratkonzentration, ist von CAIN und NiconL ${ }^{1}$ auch bereits beobachtet worden,

Die in dieser Mitteilung beschriebene Reaktion, welche von den eben besprochenen Fällen sehr verschieden ist, stellt in bezug auf die Analogie mit den Fermentreaktionen insofern einen interessanten Fall dar, als bei dieser Reaktion der Einflufs der Reaktionsprodukte (Wasser) experimentell ausgeschlossen ist, der Verlauf der Reaktion jedoch auffallende Ähnlichkeit mit den Fermentreaktionen hat. Gewisse Stheke der Kurven in den Figg. 2, 3, 4 und 6 geben diese Ähnlichkeit am besten wieder. Auch aus meinen Versuchen bei dieser anorganischen Reaktion gehen nämlich die oben (\$. 108 und 104) für die Fermente genannten Sätze mit derselben, allerdings nicht sehr grolsen Genauigkeit, gerade wie bei den Fermentreaktionen, hervor (vgl. S. 80). Abgesehen von dem vor dem Ende der Reaktion stattfindenden Ansteigen der Geschwindig. keit kann ich auch bei meiner Reaktion mit einem anorganischen Katalysator dasselbe, was Edute ${ }^{2}$ speziell nur für die Fermente behauptet hat, sagen: „Ist die Substanz (Wasserstoffsuperoxyd) im Überschusse, so ist die Reaktionsgeschwindigkeit ungefäbr proportional der Enzymkonzentration (Chromsäurekonzentration, vgl. Fig. 6, Vorderäste der Kurven $b, c, d, e)$; ist das Enzym im Überschusse vorhanden, so wird die Geschwindigkeit angenähert proportional der Substanzkonzentration" (vgl. Fig. 2, 3, 4 und 6 die letzten Äste der Kurven). Selbst wenn es sich auch später zeigen sollte, dals die der homogenen Reaktion Wasserstoffsuperoxyd-Chromsäure zugrunde liegenden Erscheinungen pritzipiell verschieden von denjenigen der Fermentreaktionen sind, mögen diese nun im heterogenen oder homogenen System verlaufen, so glaube ich doch berechtigt gewesen zu sein, diese auffallende kinetische Analogie hervorzuheben. Diese Analogie erschlittert die immer noch von manchen Autoren etwas künstlich angestrebte Unterscheidung zwischen der Wirkung der anorganischen Katalysatoren und der der Enzyme wieder an einem wesentlichen Punkte.

Erst eine ausführliche experimentelle Ausarbeitung exakt reproduzierbarer Fälle und ihre objektive theoretische Interprettierung werden zeigen, ob für die Erklärung der Fermentreaktionen

\footnotetext{
1 Journ. Chem. Soc. 83 (1903), 206.

2 Zeitschr. physiol. Chem. 52 (1907), 147.

s Sollte wohl heifsen "das Substrat".
} 
wirklich die Aufsuchung von Faktoren, welche bei gewöhnlichen Katalysatoren nicht auftreten, notwendig ist.

\section{Zusammenfassung.}

In der vorliegenden zweiten Mitteilung wurde über die kinetische Untersuchung der Reaktion Wasserstoffsuperoxyd-Chromsäure berichtet.

1. Freie Chromsäure (ohne fremde Säuren) wird durch das uberschüssige Wasserstoffsuperoxyd zum Teil zu Chromion reduziert; der ubrig bleibende Teil der Chromsäure zersetzt das Wasserstoffsuperoxyd katalytisch.

2. Der nach Ablauf der Reaktion reduzierte Teil der Chromsäure (ca. $28 \%$ ) ist unabhängig von der angewandten Anfangskonzentration des Wasserstoffsuperoxyds und nahezu unabhängig von der Konzentration der Chromsäure (Tabelle 1).

3. Die katalytische Zersetzung des Wasserstoffsuperoxyds verlauft mit einem bis jetzt nicht bekannten, komplizierten, experimentell aber genau reproduzierbaren Zeitgesetze: so lange das Wasserstoffsuperoxyd im Überschusse vorhanden ist $\left(C_{\mathrm{H}_{3} \mathrm{O}_{3}}>10 C_{\mathrm{CrO}_{3}}, C_{\mathrm{CrO}_{2}}=\right.$ etwa 0.001 bis $0.005 \mathrm{Mol}$. pro Liter $)$ ist die Geschwindigkeit seiner Zersetzung (die pro Zeiteinheit entwickelte Sauerstoffgasmenge) sehr wenig abhängig von der Zeit resp. von der vorbandenen $\mathrm{H}_{2} \mathrm{O}_{2}$-Konzentration, indem die Geschwindigkeit nur ungefähr proportional der dritten Wurzel aus der abnehmenden Wasserstoffsuperoxydkonzentration abnimmt. Dann aber, wenn nur ca. $10 \mathrm{mal}$ mehr Mole $\mathrm{H}_{2} \mathrm{O}_{2}$ als Mole angewandter katalysierender Chromsäure noch vorhanden, wenn etwa $90-85 \%$ der angewandten $\mathrm{H}_{2} \mathrm{O}_{2}$-Menge schon zersetzt sind, steigt die Geschwindigkeit sehr schnell auf das zwei- bis dreifache, um nach einem sehr scharfen Maximum ebenso schnell auf Null zu fallen, was mit der vollständigen Zersetzung des Wasserstoffsuperoxyds zusammenfällt (Fig. 1).

4. Bei einem Überschusse von Wasserstoffsuperoxyd und konstanter Menge Chromsäure ist der relative Umsatz in gleichen Zeiten um so kleiner, je grôfor die Anfangskonzentration des Wasserstoffsuperoxyds war (Fig. 2, Tab. 2).

5. Die Konzentrationen des im Reaktionsgemische noch vorhandenen Wasserstoffsuperoxyds, bei welchen die Reaktionsgeschwindigkeit gegen Ende der Reaktion das Minimum und gleich darauf das Maximum bildet, sind bei gegebener Chromsäure- 
menge ganz bestimmte, von der Anfangskonzentration des Superoxyds unabhängige Punkte.

6. In jedem Momente der Katalyse ist ihre Geschwindigkeit bei konstanter Chromsäurekonzentration durch die im Reaktionsgemische vorhandene Wasserstoffsuperoxydkonzen. tration eindeutig bestimmt, wenn auch die gegebene Menge Chromsäurelösung schon mehreremale mehr oder weniger Wasserstoffsuperoxyd bis zu Ende zersetzt hatte (Figg. 3, 4 and 5).

7. Bei verschiedenen Chromsäurekonzentrationen gilt:

Die Geschwindigkeit im nahezu horizontalen Teile der Kurve (Geschwińdigkeit als Funktion der $\mathrm{H}_{2} \mathrm{O}_{2}$-Konzentration) ist ungefähr proportional der angewandten Chromsäuremenge.

Die noch rorhandene $W$ asserstoffsuperoxydkonzentration, bei welcher das Minimum (unscharf) resp. Maximum der Geschwindigkeit auftritt, ist ungefähr proportional der angewandten Chromsäurekonzentration (Fig. 6, Tabelle 3).

Auch bei versohiedenen Chromsäurekonzentrationen ist die Geschwindigkeit in jedem Augenblicke aufser durch die angewandte Ghromsäuremenge nur'durch die vorhandene $\mathrm{H}_{2} \mathrm{O}_{2}$-Konzentration vollständig definiert (vgl. 6).

8. Zwischen der in der ersten Mitteilung beschriebenen monomolekularen $\mathrm{H}_{2} \mathrm{O}_{2}$-Katalyse durch das Dichromat und dem hier mitgeteilten merkwurdigen Zeitgesetze der Katalyse mit freier Chromsăure läst sich auf verschiedene Weise der Übergang experimentell realisieren.

9. Die Eigentümlichkeiten der Katalyse WasserstoffsuperoxydChromsäure werden vor der Hand am plausibelsten, wenn man die Reversibilität der eventuellen momentanen Änderungen im Zustande des Katalysators unter dem Einflusse verschiedener Mengen des vorhandenen Wasserstoffsuperoxyds mit Hilfe von $\mathrm{Z}$ wischenverbindungen annimmt.

10. Diese Änderungen und dadurch auch der Reaktionsverlauf sind ăufserst empfindlich gegen $\mathrm{H}^{\cdot}$ bzw. OH'-Ion. Man könnte die Katalyse des Wasserstoffsuperoxyds durch Chromsäure und Chromate als Indikator bei der Titration einer Säure mit Alkali benutzen (Tabelle 4, S. 102).

11. Die Katalyse Wasserstoffsuperoxyd-Chromsäure zeigt in ihrem kinetischen Verlạfe gewisse auffallende Analogien zu 
den sogenannten Fermentreaktionen, worauf auch entsprechend hingewiesen wurde.

Die Untersuchung dieser Katalyse unter Aufsuchung analoger Fälle wird fortgesetzt.

Auch bei dieser Gelegenheit möchte ich Herrn Prof. G. Bredig fur die Anregung und freundliche Unterstutzung bei dieser Arbeit meinen innigsten Dank aussprechen.

Heidelberg, Chemisches Universitätslaboratorium.

Bei der Redaktion eingegangen am 29. August 1907. 